\title{
Automated grey box model implementation using BIM and Modelica
}

\author{
Ando Andriamamonjy ${ }^{1,2}$, Ralf Klein ${ }^{2}$, Dirk Saelens ${ }^{1,3}$ \\ ${ }^{1}$ KU Leuven, Department of Civil Engineering, Building Physics Section, Kasteelpark Arenberg 40 box 2447, BE-3001 Heverlee, Belgium \\ ${ }^{2}$ KU Leuven, Technology Cluster Construction, Technology Campus Ghent, Gebroeders De Smetstraat 1, BE-9000 Ghent, Belgium \\ ${ }^{3}$ EnergyVille, Thor Park 8310, BE-3600 Genk, Belgium
}

\begin{abstract}
A large part of energy usage in buildings occurs during the operational phase, emphasising the need for efficient and improved facility management, operation and control. Model Predictive Control (MPC) or Fault Detection and Diagnosis (FDD) are among the strategies that allow minimising energy use and costs during operation. However, the need for fast and accurate dynamic models (e.g. grey box model), which are time-consuming and challenging to implement, precludes their systematic integration in the built environment. A typical grey-box modelling approach consists of manually implementing several grey-box model structures with an increasing level of complexity before performing a forward selection procedure to identify the optimal configuration. The link between the different grey-box models and the monitored data is also established manually. Such an approach can be both time-consuming and error-prone and involves a significant cost that hampers the broad adoption of strategies such as MPC and FDD.

This study proposes a tool-chain that uses BIM to automatically generate several grey-box structures with added complexities stemming from the specific geometry and design of the building. More specifically, an existing rule-based IFC to Modelica interface is extended to automatically create several Modelica-based grey box models that gradually take into account the building's specific information and characteristics. Additional rules are also proposed to automate the connection between the models and the building monitoring system.

As a forward selection approach, a multi-objective optimisation using the NSGA-2 algorithm is adopted. The application of the tool-chain on two case studies shows that the integration of BIM to automate the implementation of grey box models, not only reduces the human involvement in the modelling process but can also produce more accurate models. Besides, this study shows that the use of multi-objective optimisation with datasets from two different seasons results in models that are valid for all seasons.
\end{abstract}

Keywords: Building Information Model, grey box model, Multi-objective optimisation, Fault detection and diagnosis, Building envelop

\section{Contents}

\section{Introduction}

\section{Motivation}

3 Context and Methodology outline

3.1 Context ................. 4

3.2 Methodology outline .......... 4

\section{Preconditions}

\section{Automated grey box structure diversification and se-} lection

5.1 BII description and limitations for grey box modelling ................. 7

5.2 Topology diversification ......... 8

5.3 Automated grey box model implementation . . 9

6 Automated data acquisition for model training 9

6.1 Construction stage: BMS configuration . . . . 11

6.2 Operational stage ............ 11

7 Model selection using multi-objective optimisation 11

18 Case studies and experiment setup $\quad 12$

8.1 Case 1: Fictive building . . . . . . . . . . 12

8.2 Case 2: Existing building . . . . . . . 13

9 Results and interpretation 13

9.1 Case 1: Fictive building . . . . . . . . . . . . 14

9.2 Case 2: Existing building . . . . . . . . . . 16

9.2.1 Small FDD example . . . . . . . 16

10 Discussion 16

7

11 Conclusion

18

\section{Introduction}

The recent emergence of small, low-cost and wireless sensors supports the collection of monitored data in the built environment [1]. This technological progress combined with the capability to predict buildings' dynamic behaviour offers several en- 
ergy savings opportunities. For instance, dynamic building energy models can be integrated into advanced and cost-effective control strategies such as Model Predictive Control (MPC) or Fault Detection and Diagnosis (FDD). Avci et al. [2] applied MPC for HVAC load control leading to a cost reduction of $13 \%$ and a decrease of $23.6 \%$ of the energy use during peak hours. Similarly, Pedersen et al. [3] proposed MPC scheme that can increase cost savings by $6 \%$ after the building's retrofit and reduce the $\mathrm{CO}_{2}$ emissions by $3 \%$. Likewise, Jorissen [4] developed a user-friendly, object-oriented optimal control approach which can reduce operation costs by up to $65 \%$. Furthermore, dynamic models can be used to detect occupants' energy inefficient behaviour and defects in indoor equipment (e.g. faulty sensors) [5]. These models can also be used for building characterisation during which parameters such as the UA-value are estimated [6, 7].

Dynamic models originate from three different modelling approaches in which accuracy and -minimal- computation time are keys for the use in MPCs and FDDs strategies.

1. The building energy models used during the design phase seem to be an obvious choice since they are readily available at the operational phase. However, this type of model, commonly referred to as white box model, is detailed and can induce a significant computation time. It is consequently incompatible with strategies requiring a fast response. Also, uncalibrated white box models often show a certain discrepancy with the actual performance $[8,9,10,11]$. In some cases, the actual energy use can correspond to the double of the prediction [9]. Consequently and considering the large number of parameters of such models, a rather difficult and time-consuming calibration task is necessary [12].

2. Another modelling approach relies on past measured data. This method referred to as black-box modelling provides a fast response dynamic model. Its application is relatively straightforward but requires a significant amount of data to be properly trained. Another drawback is the absence of physical meaning to black-box model parameters, thus limiting its use for building characterisation (e.g. in Ref $[13,14,15,16])$.

3. As a trade-off approach, a grey-box model represents the building with a network of resistances and capacities $(\mathrm{RxCy}) . \quad x$ represents the number of resistances and $y$ the number of capacities (model order). A grey-box model combines information from measured data with prior knowledge of the building structure, generating a simplified dynamic model with a fast response. Grey box models are commonly used for MPC as well as for building characterisation $[6,7,1]$.

This study focuses on the development of a technical (software application) approach that automates the implementation of grey box models, especially for a future application for MPCs or FDDs.

As reported by Killian et al. [17], the effort to construct the model and its integration in the Building Management System (BMS) constitute one of the major caveats for the broad adoption of such strategies (MPC or FDD). They emphasise both the need for and the lack of a tool to automate the implementation of these strategies and pinpoint the necessity of an efficient modelling approach combined with a building-specific MPC scheme. Furthermore, De Coninck et al. [1] reported that the implementation and selection of the adequate grey-box model represent $70 \%$ of the effort required to develop an MPC controller, further emphasising the need for an automated strategy.

This modelling effort is necessary since there is no RC-structure ( $\mathrm{RxCy}$ ) that can produce the best prediction for any typology and configuration of buildings, although second order models $(\mathrm{RxC} 2)$ are accepted as the simplest representation of a building zone $[18,19]$. As it will be shown in section 2, several authors indicate that second order models rarely provide accurate predictions. In practice, more complex, case-specific and diverse grey box structures ( $\mathrm{RxCy}$ ) are preferred.

To identify the best $\mathrm{RxCy}$ structure representing a specific building (or zone), a typical approach relies on a forward selection procedure during which several fixed RC structures with increasing complexities are defined. The added complexity is often related to the geometry and specific design of the considered building as well as the representation of building elements in terms of $\mathrm{RC}$ components (e.g. walls represented by $\mathrm{R} 1$ or R2C1). The selection starts from the simplest structure (e.g. RxC2) and evolves to a more complex one that can satisfy the different validation criteria. Furthermore, the optimisation algorithms used for the selection procedure often require valid initial values retrieved from the physical charachteristic of the actual building (e.g. in Ref. [20, 21, 22, 7]). Consequently, a prior knowledge of the building characteristics is necessary either to implement the set of different grey box structures ( $\mathrm{RxCy}$ ) from which the optimal one is drawn or to compute adequate initial values of the model parameters.

The information required to implement different $\mathrm{RxCy}$ structures tailored for a specific building and to generate adequate initial values can be deduced from the emerging technology [23] in the Architecture Engineering and Construction (AEC) industry: Building Information Model (BIM). A BIM is a digital representation of physical and functional characteristics of a building project. It includes 3D geometry, spatial relationships, geographic information, wall and windows orientations and building element properties [24, 25, 26].

Recently, the integration of BIM with energy simulation has gained a lot of attention. In that regard, several studies (e.g. in Refs. [27, 28]) have investigated the combination of simulation engines with proprietary BIM tools either through a direct integration or through the BIM tool's Application Programming Interface (API). Other alternative approaches (e.g. in Refs. [29, 30] or in commercial tools such as DesignBuilder ${ }^{\mathrm{TM}}$ ) rely on the open, straightforward and de-facto industry standard schema Green Building XML (gbXML) to overcome the dependence on a specific BIM tool. Likewise, several research studies ( e.g. Refs. [31, 32, 33, 34, 35]) have developed different approaches that integrate the openBIM format 
IFC (Industry Foundation Class) with different energy simulation engines. The IFC (Industry Foundation Class), developed by the buildingSMART association, is currently the only nonproprietary, standard (ISO 16739) BIM format. It is an objectoriented data model that can support the information exchange required throughout the buildings' life cycle [36]. The use of IFC ensures interoperability between different BIM tools used by stakeholders [37] as well as the independence towards specific BIM application vendors.

Independently of the BIM format adopted, the reliance on traditional simulation engines such as EnergyPlus, TRNSYS or DOE-2 constitutes a common point among most of the existing BIM to energy simulation approaches. These simulation engines are mostly compatible with the detailed design phase and lack modularity and flexibility [38, 39].

The recent interest towards the modular, flexible, equationbased simulation engine Modelica is driven by the need to overcome the limitations of these traditional simulation engines $[38,39]$.

The first open, semi-automated and standardised approach coupling IFC and the modelling language Modelica has been developed under the umbrella of the IEA EBC Annex 60 project [40]. The approach adopted uses SimModel [41] as a pivotal data model between IFC and Modelica. While recently, Andriamamonjy et al. [42] have developed another method that permits a direct translation of IFC into a Modelica model. These existing IFC to Modelica approaches generate only one level of complexity of a model, which is compatible with the detailed design phase. Consequently, a method allowing the use of IFC information to create lumped RC structures with several levels of complexity in Modelica is still missing.

The research presented in this paper demonstrates the relevance and applicability of a BIM (IFC) based workflow for the automation of the development, training and selection of grey box structures ( $\mathrm{RxCy})$. The software development hereby presented uses IFC to reduce the need for manual configuration when implementing grey box models. The strategy adopted relies on a BIM to automatically generate several grey box structures ( $\mathrm{RxCy}$ ) with increasing complexity and streamline the entire model selection process. The objective of this study can be summarised within the following points:

1. The openBIM format IFC4 is used in combination with the Modelling language Modelica to automate the generation of a set of grey box Modelica models. In order to relieve modellers from the manual task of developing the different grey box structures, from which the optimal model is chosen in a forward selection approach, the here-presented approach takes advantage of BIM to generate grey box structures that mirror the specific design of the actual building and compute the initial values.

To do so, an earlier developed, rule-based IFC to Modelica approach (BII) [42] is extended and improved to enable the automated implementation of several (multi or monozone) grey box structures with different levels of complexity. It relies on Modelica's component-oriented characteristic and BII's flexibility to produce several grey box struc- tures that gradually take into account the different specificities of the considered building (e.g. HVAC equipment, orientation, adjacent zones).

2. Furthermore, this study shows how BIM can be used to automate the acquisition of the training data from the Building Monitoring System (BMS). It also presents a workflow demonstrating the potential use of the automated grey box modelling approach.

3. To demonstrate the advantages and potential benefits of such an automated workflow, a multi-objective optimisation approach is used for the model selection process.

Section 2 is a literature review that further emphasises on the reasons and motivations behind this work. The context of use and the general outline of the automated approach are explained in section 3, and section 4 details the BIM modelling conditions necessary to adopt the present automated grey box modelling approach. The technical basis for the automated implementation of the different grey box structures from a BIM is discussed in section 5 .

First a short presentation of BII [42] and its limitations in term of grey box modelling are explained in section 5.1. Then, the improvements brought to BII and the automated grey box modelling process are discussed in sections 5.2 and 5.3. The implementation of the link between the Modelica grey box models and the Building Monitoring System (BMS) is given in section 6. Finally, the forward selection procedure that uses a multiobjective optimisation is presented in section 7 .

The toolchain has been applied in a virtual scenario and on a real -in use- lecture room of a university building (see section 8) using a typical occupancy schedule of school buildings or lecture rooms. The achieved results in both case studies are shown in section 9 which also illustrates the potential of the toolchain by identifying a manually induced offset error in an indoor temperature sensor.

\section{Motivation}

Although a second-order model $(\mathrm{RxC} 2)$ is known as the simplest representation of a building zone [18, 19], this section shows through a literature review that, in practice, more complex, case-specific and diverse grey box structures ( $\mathrm{RxCy})$ are preferred.

For instance, Bacher et al. [22] evaluated ten grey box structures with a level of complexity varying from second to fifth order. The structures differ based on the representation of the building elements in term of RC components. As an example, some structures describe the building envelope with a simple resistance (R1) while others take into account the thermal mass and depict the envelope with two resistances and one capacity (R2C1). Also, some RC structures consider a heater component as a simple heat input while others try to include dynamics by using one resistance and one capacity (R1C1). Bacher et al. [22] found that a fourth order model (R4C4) depicting both sensor and heat input with a R1C1 component yields the best results and satisfies the validation criteria with the dataset used. 
Similarly, Berthou et al.[18] evaluated four model structures and came to the conclusion that a more complex third-order model (R6C3), which better simulates the exchange between the furniture and air capacitance, provides better results especially during the winter. In addition, Hedegaard et al. [6] show that a R4C3 model gives better results than the conventional $\mathrm{R} 3 \mathrm{C} 2$, however underlining the case-specific nature of the assumptions they have adopted.

Additionally, Reynders et al. [21] observed that a fourth order (R6C4) model in which the effect of the heat exchange through the ground are taken into account, produces optimal results for a typical uninsulated detached Belgian house. For insulated buildings, a fifth order (R8C5) model that additionally includes a representation of the roof yields better accuracy. De Coninck et al. [1] reach a similar conclusion considering that the integration of the exchange with the adjacent zones significantly improves the accuracy of their models. Reynders et al. [21] also investigate the impact of different sets of input data on the model's accuracy and found that the integration of solar radiation based on window orientation has little effect. In contrast, De Coninck et al. [1] found that the integration of oriented solar radiation contributes to a large reduction of model errors. Wang et al. [43] even advocate that, in addition to the windows, external walls should be considered according to their orientations. Furthermore, most optimisation algorithms used for the identification process include the interior point algorithm $[18,1]$ and the maximum likelihood estimation [20, 21, 22, 7] (using the CTSM-R package [44]). These algorithms require valid initial values which are calculated from the building characteristics.

The diversity of grey box structures found in literature and the need for adequate initial values emphasise the fact that the bestselected RC-structure is (potentially) case dependent. The potential impact of the building's geometry and design must then be considered because a model chosen from a fixed set of RCstructure might overlook some effects specific to a case and, thus jeopardising the accuracy of the accepted model. Therefore the set of RC structures, from which the final RC model has to be chosen, needs to include RC-structures that consider the actual building's window orientation, wall orientation, adjacent zones and systems. However, collecting such building information manually and implementing a set of grey-box structures with increasing complexities can be time-consuming and errorprone.

Consequently, this study proposes a flexible technical approach to source building information from IFC and automatically generate a set of grey box model structures with increasing complexity; from which the best $\mathrm{RxCy}$ structure is drawn. Also, it uses BIM to establish the link with the monitored data stored in a Building Monitoring System (BMS).

\section{Context and Methodology outline}

\subsection{Context}

As stated earlier, BIM is a data-rich, object-oriented representation of the physical and functional characteristics of a building. It serves as an information source to improve the collaboration between different stakeholders. Its interoperability capabilities permit a seamless data transfer from one application to another throughout the entire building lifecycle stages, thus reducing redundancy and data duplication. Furthermore, the use of a BIM can ensure a better building design by reducing errors, omissions and rework and by allowing the client to get involved and get a better understanding of the project [45, 46, 47, 48, 49].

In this study, BIM is used as a source of information to construct a set of grey box structures with increasing complexities. BIM includes the characteristics of wall layers and material properties (thermal conductivity or density) from which the initial values for the optimisation algorithm can be computed. BIM also comprises wall and windows orientation as well as space configuration which are used to vary the structure of grey box models.

Besides, driven by the overall potential benefits of BIM, the worldwide adoption of BIM-based workflows has increased over the past decade. A 2010 survey conducted by the McGraw Hill Construction [49] reported that $36 \%$ of the professionals from western European countries participating in the study [49] have been using BIM in their projects. Since then, the BIM adoption rate has increased. A recent 2015 survey from the same institution predicts on average $95 \%$ more BIM users worldwide within the next two years [46]. This increase is further accentuated in the United Kingdom (UK) by the government mandate (April 2016) that imposes the adoption of BIM by all publicly funded projects. A 2017 survey from the National Building Specification (NBS- UK -) also reported that $60 \%$ of the contractors (from the UK) use BIM during the design phase of a project and this number is expected to reach $95 \%$ in the next three years [48]. A wider adoption of BIM is also noted in other parts of the world. For instance, in North America, its use has surged from $49 \%$ in 2010 to $71 \%$ in 2012 among the different building construction actors (Contractors, Architects and engineers) [47]. A similar growth rate is still expected today. In China, the expansion rate is predicted to reach more than $100 \%$ [46].

This recent interest towards BIM use and adoption can be leveraged to facilitate the integration of energy saving strategies such as MPC and FDD.

\subsection{Methodology outline}

Figure 1 shows how the proposed workflow is integrated within the design and building operation processes. This study assumes that BIM is used throughout the different building lifecycle stages and the openBIM format Industry Foundation Class (IFC4) is adopted as the exchange format between the stakeholders.

During the design phase (figure 1; design phase), the building design team implements a BIM that shall satisfy certain preconditions (see section 4) especially for the description of the building envelope. This step ensures that the relevant information required to generate the $\mathrm{RC}$-structures is correctly included in BIM (section 4).

Subsequently, at the operational phase (figure 1; operational phase), BIM (IFC file) is used as input to an automated toolchain which integrates the automated grey box modelling 


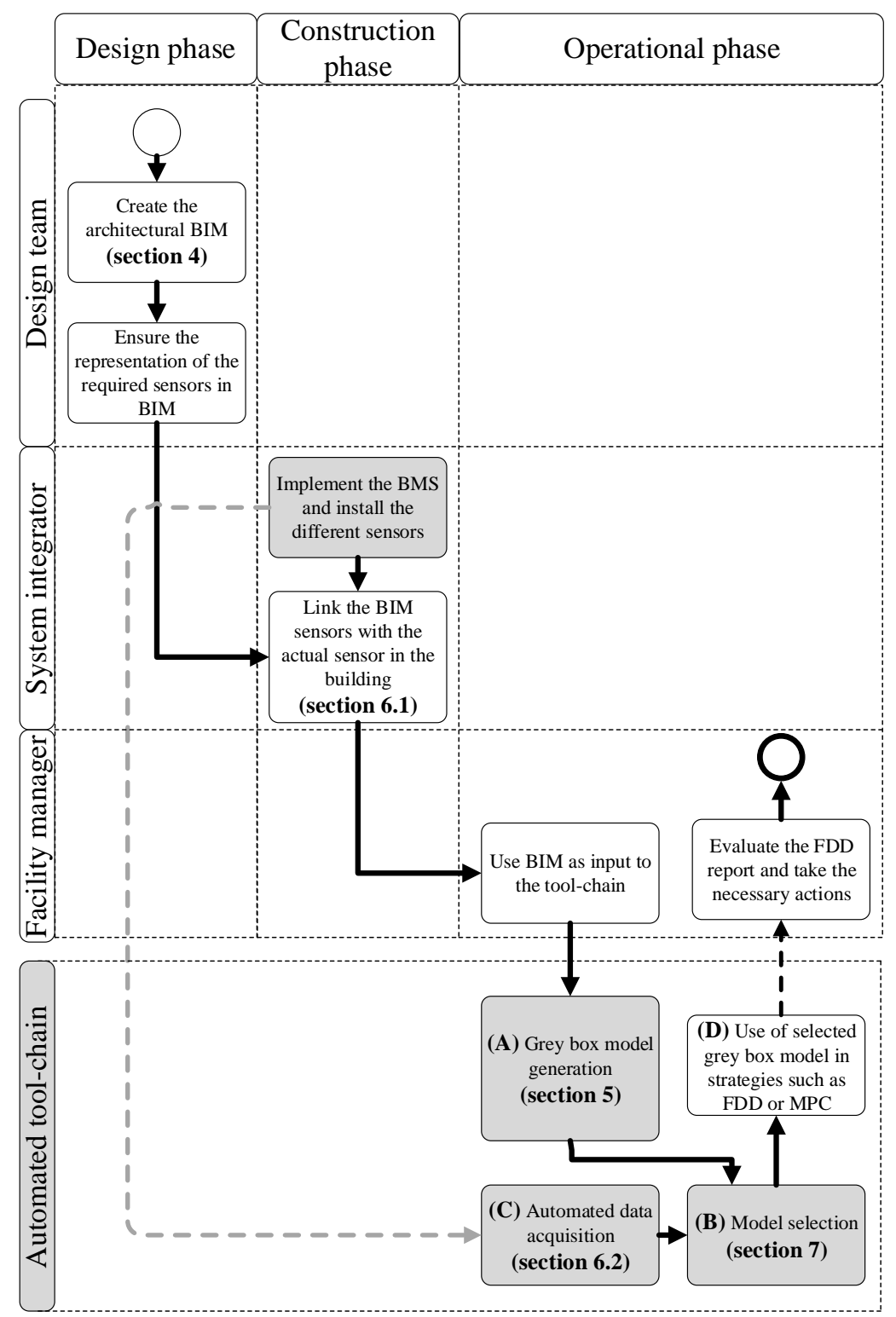

Figure 1: Overview of the integration of the automated workflow throughout the different building life cycle stages

approach. The former generates a set of RC-model structures using the physical data contained in BIM (figure 1.A). A selection procedure (figure 1.B) combined with an automated data acquisition technique (figure 1.C) then allows identifying the best RC structure. At last, the best-selected model can be integrated into energy-saving strategies such as FDD or MPC (figure 1.D). More specifically (see figure 1):

(A) Section 5 details the underlying technical approach allowing to implement different RC structures from BIM. It shows the current limitations of BII [42] and the improvement brought to the latter to enable the development of RC-structures with different levels of complexity.

(B) The generated RC structures can be integrated into existing forward selection approaches (e.g. in Ref [1]). How- ever, in section 7 , the use of a multi-objective evolutionary algorithm for the parameter estimation process is investigated.

(C) Model selection and parameter estimation require access to measured data. Section 6 shows how BIM can be leveraged to automatically retrieve sensor data from a Building Monitoring System (BMS). A short overview of existing approaches that integrate BIM with real-time data is given followed by the implementation adopted in this study.

(D) The present automated grey box modelling approach can be combined and integrated with existing MPC or FDD techniques. For instance, it can be used to facilitate or even automate the integration of the MPC technic for building heating systems proposed in Ref. [50]. It can also help 
support the broad integration of the Economic MPC as defined by Zong et al. [51]. However, developing a novel FDD or MPC approaches is out of the scope of this study.

Detailed explanations of the proposed methodology are provided in section 4 to section 7 .

\section{Preconditions}

This study assumes that a BIM is available at the operational phase either from 3D-reconstruction for existing facilities or from the design phase for new buildings. The openBIM format Industry Foundation Class (IFC4) is adopted as the exchange format between the stakeholders. IFC is an objectoriented data model in which an object is referred to as entity (e.g. IfcSpace or IfcWallStandardCase). The package of information describing the relationship between the entities is called concept (e.g. the Space boundaries concept defines how a space entity -IfcSpace- is related to a wall entity If cWallStandardCase-). A complete description of the IFC entities and concepts can be found in the publicly available IFC4 specifications [52].

In the context of this paper, it is assumed that the difference between the BIM and the actual building is negligible especially regarding the geometry of the building. The building envelope representation shall comply with the following rules:

1. Spaces shall be defined and fully enclosed within constructions. The Space boundaries [52] concept shall be defined and exported. If applicable, spaces (IfcSpace) should be assigned to a zone entity (IfcZone).

2. Material properties (especially thermal conductivity, specific heat capacity and density) should be included according to the Material Layer Set or Material Layer Set Usage concepts [52].

3. Entities contained in a space shall be related to the space entity (IfcSpace), in which they are contained, through the Spatial Containment concept.

4. A temperature sensor object (IfcSensor) shall be placed inside each space (or room) and should be described as presented in table 1. The parameter PredefinedType must be equal to TEMPERATURESENSOR while Pset_SensorTypeTemperatureSensor. TemperatureSensorType should be set as ROOMTENPERATURE. Similarly, as shown in table 1, an outdoor temperature sensor and a solar radiation sensor must be represented on the BIM.

5. An actuator object (IfcActuator) shall be attached to each BIM object representing a controllable system component (e.g. IfcSpaceHeater).

6. A Pset_DataBase property set must be defined for each If cActuator or If cSensor entity. Pset_DataBase is a custom property set proposed in this study to contain the information related to the actual sensor represented by an
Table 1: Minimum information describing an IfcSensor entity

1) Room temperature sensor

PredefinedType:TEMPERATURESENSOR

Pset_SensorTypeTemperatureSensor.TemperatureSensorType: ROOMTEMPERATURE

\section{2) Outside temperature sensor}

PredefinedType:TEMPERATURESENSOR

Pset_SensorTypeTemperatureSensor.TemperatureSensorType: OUTSIDETEMPERATURE

\begin{tabular}{l}
\hline 3) Solar radiation sensor \\
\hline PredefinedType:USERDEFINED \\
Pset_SensorTypeSolarRadiationSensor.SolarRadiationSensorType: \\
GLOBALRADIATION
\end{tabular}

IfcSensor entity. Pset_DataBase is to be filled by the system integrator at the operational phase.

It comprises three parameters:

- Description: It provides a generic description of the sensor

- DbType: Information related to the type of database used by the BMS.

- ColumnId: Identifier of the database column in which the measured value is stored.

Table 2: Proposed property set attached to If cSensor or If cActuator entities, used to link BIM and BMS

\begin{tabular}{ll}
\hline Pset_DataBase & \\
\hline Description & P_SINGLEVALUE /IfcText \\
DbType & P_ENUMERATEDVALUE / IfcLabel / \\
& PEnum_DatabaseType: RELATIONAL, OTHER \\
ColumnId & P_SINGLEVALUE /IfcIdentifier \\
\hline
\end{tabular}

Figure 2 represents a fictive building whose BIM is designed according to the previously defined guidelines. The building is a simple detached house with two rooms (see figure 2.II) defined as space entities (IfcSpace). The size of each room as well as the orientation and physical properties of the construction elements (walls, roof, floor and windows) are equal to those of the BESTEST case 900 [53]. Electrical heaters (figure 2.b) are placed in all rooms. Each of them is logically connected to an actuator (IfcActuator, see figure 2.d) through the Element connectivity concept. These actuators are in turn connected - through the Port connectivity concept- to a controller object (IfcController, see figure 2.c). BIM objects representing temperature sensors (IfcSensor, see figure 2.a) are connectedthrough the Port connectivity concept- to the controllers and placed in each room.

The BIM presented in figure 2 will be used as an example to illustrate the different modifications and improvements brought to BII for the purpose of grey box modelling. 


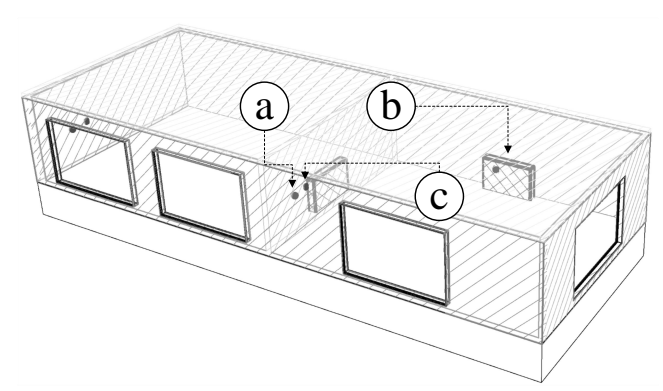

I.

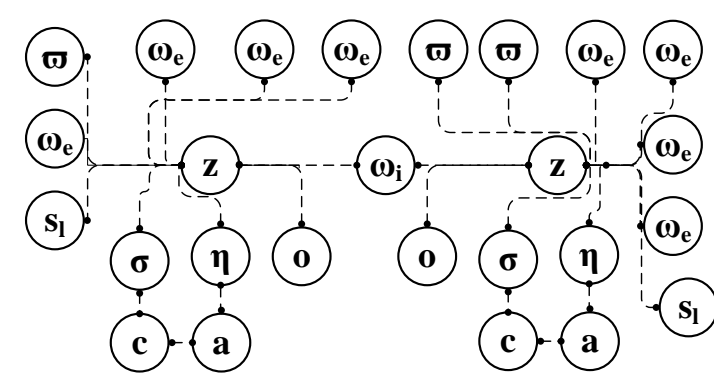

III.

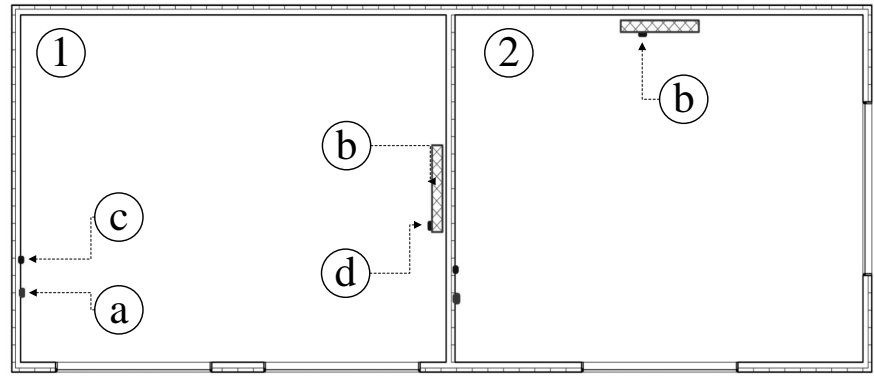

II.
(๘) Windows (IfcWindow)

(1) External constructions

e (IfcWallStandardCase, IfcSlab, IfcRoof, IfcDoor

Boundary constructions

$\omega_{\mathrm{i}}$ (IfcWallStandardCase, IfcSlab,
IfcRoof, IfcDoor)

(o) Occupancy

(S) Slab (IfcSlab) (z) Zone (IfcSpace-IfcZone)

(б) Sensors (IfcSensor)

(c) Controller (IfcController)

(1) Heaters

ワ) (IfcSpaceHeater)

(a) Actuator

Figure 2: 3-D view of the BIM that represents a fictive two-zone building used for illustration purpose. (I) 3D view of the building rendering the different equipment contained in the zones: (a): temperature sensor, (b): heater, (c): controller, (d): actuator. (II) Floor plan. (III) Topology generated with the current version of BII [42].

\section{Automated grey box structure diversification and selec- tion}

\subsection{BII description and limitations for grey box modelling}

BII, as described in [42], is a BIM-to-energy-simulation interface that is capable to translate geometry, systems and control information in BIM into a detailed building energy simulation model implemented in Modelica. This section presents BII's limitations precluding its use for grey box modelling.

BII consists of two main parts: the topology generation and the mapping process.

- The topology generation is a rule-based process that converts IFC into a simplified structure (called topology) compatible with a direct mapping into Modelica [42]. A topology revolves around two components: nodes and edges. A node carries the same information (e.g. Property set) as the specific IFC entity it represents but it can be directly mapped into a particular Modelica component. As an example, IFC entities that represent physical elements (e.g. walls) are converted into nodes. Edges, on the other hand, describe specific IFC concepts (e.g. space boundary concept) that are to be translated into Modelica connections. The combination of both nodes and edges results in a data structure directly convertible into a Modelica model. The different conversion rules are thoroughly explained in [42].

The complexity of the topology generated in [42] is designed to be compatible with a detailed BEPS modelling. As an example, figure 2.III shows the topology generated for the BIM in figure 2.I and figure 2.II. For each space, external walls are modelled regarding their azimuth, i.e. entities representing external walls are converted into nodes by considering their orientation. The orientation of the windows is equally relevant. Consequently, if a grey-box model is generated, assuming each wall node $(\omega)$ is represented by two resistances and one capacitor (R2C1), this topology (figure 2.III) results in an $11^{\text {th }}$ order, two-zone grey-box model. Such complex and over-parametrized model can be over-fitted. Furthermore, BII currently generates one topology (figure 2.III), thus limiting the choice of RC structure for the forward selection approach. Additional rules then need to be added to the topology generation process to create additional topologies as presented in section 5.2 .

- The mapping process translates a topology into a Model- 
ica model by converting nodes into Modelica components and edges into Modelica connections (Modelica statement connect).

The correspondence between nodes and components is stored in a XML-file called Library template (e.g. in Table 3). This Library template contains a set of component templates which individually contains the description of a Modelica component corresponding to a node (e.g. in Table $3,1^{\text {st }}$ row, the zone node $(z)$ is associated with a one-capacity Modelica component).

Section 5.2 shows the different rules added to the topology generation process to create additional topologies compatible with grey box models.

\subsection{Topology diversification}

As stated earlier, the original topology generation process can generate only one topology, thus precluding the use of BII for grey box modelling. Figure 3 presents eight (8) topologies with different degrees of complexity that were added to BII for the purpose of grey box modelling. The different structures vary from mono-zone to multi-zone and are detailed as follows.

1. Topology 1 (e.g. figure 3.1) is the simplest because: (1) it represents a space (IfcSpace) as a node (Z), (2) merges all the external walls (related to the space) into one node $\left(\omega_{e}\right)$ regardless of their orientation, (3) merges all the windows connected to the space into one node $(\bar{\omega})$ regardless of their orientation and lastly (4) ignores the internal walls. Furthermore, if several spaces are related through a zone entity (IfcZone), then a node $(\kappa)$ representing the capacity of the internal walls is added (figure 3.1 represents the topology 1 of the room 2 in figure 2.I).

For each node, the aggregated resistance $R_{\text {total }}$ and capacity $C_{\text {total }}$ are computed from the properties of the construction entities using respectively the equations 1 and 2 .

$$
R_{\text {total }}=\left(r_{s i}+r_{s o}+\sum_{i=1}^{L} \frac{x_{i}}{k_{i}}\right) / A_{\text {total }}
$$

Where $x_{i}, k_{i}$ are respectively the thickness and the thermal conductivity of the $i^{\text {th }}$ layer, $A_{\text {total }}$ the overall area of the construction element. $r_{s i}$ and $r_{s o}$ are the inside and outside surface convection resistance.

$$
C_{\text {total }}=A_{\text {total }}\left(\sum_{i=1}^{L} x_{i} \cdot \rho_{i} \cdot c p_{i}\right)
$$

$\rho_{i}$ and $c p_{i}$ designate the density and the specific heat capacity of the layer $i$.

The values of $C_{\text {total }}$ and $R_{\text {total }}$ will be used to compute the initial values in the mapping process (see subsection 5.3).

Entities (e.g. heaters (IfcSpaceHeater) and sensors (IfcSensor)) that are contained in a space (IfcSpace) are represented with a node $(\operatorname{resp} .(\eta),(\sigma))$. If a space contains several air terminals (IfcAirTerminal) including at least one supply air terminal and one return air terminal, then a ventilation node $(\alpha)$ is added to the topology. Lastly, an additional node $(O)$ representing the occupancy is added to the topology. Figure 3.1 presents the topology 1 generated for room 2 of the example case (figure 2).

2. Topology 2 adds a node ( $\iota$ ) that represents the infiltration to topology 1. Figure 3.2 illustrates the representation of topology 2 for room 2 (see figure 2).

3. Topology 3 is an extension of topology 2 and takes into account the orientation of the windows. As a result, windows with different orientations are represented with different nodes $\left(\left(\bar{\omega}_{1}\right),\left(\bar{\omega}_{2}\right)\right.$ (see figure 3.1). The windows' azimuths (azi) are computed based on the angle between the (true) north and a vector normal to the base surface of the bounded space. This normal vector is computed from the Axis 2D Geometry and Product Placement concepts [42]. Also, the building's geographical information (longitude and latitude) are extracted from the IfcSite entity. This information serves to compute the solar radiation components corresponding to the orientation. Figure 3.3 describes the representation of topology 3 for room 2 .

4. Topology 4 includes the heat exchange with the boundary zones, meaning that the internal walls are represented by nodes $\left(\omega_{i}\right)$. Topology 4 can be based on any of the previously described topologies. As an example, a topology 4-1 (see figure 3.4) is a topology 1 to the exception that it considers the boundary connection with the neighbouring zone 1 (see figure 2).

5. Topology 5 considers the difference of thermal properties between the different external constructions. For instance in figure 2, the roof does not have the same construction properties as the external walls. In such case, they are represented by two distinct external wall nodes $\left(\omega_{e}\right)$ (see figure 3.5).

6. Topology 6 considers additional edges between the external walls and the different heat inputs in the model (e.g. Solar radiation) to acknowledge the radiative gains within the walls.

7. Topology 7 can be based on one of the topologies 1 to 4 , the difference being that it considers the walls' orientation (see figure 3.7).

8. The previous topologies can be extended into multi-zone models by duplicating the chosen topology for each space or zone in the BIM. For instance, figure 3.8 shows the topology 1-multizone of the zones 1 and 2 in figure 2 .

Several other topologies can be generated through a combination of these 8 basic patterns. Subsequently, they are transferred to the mapping process to generate the different Modelica models. 
1)

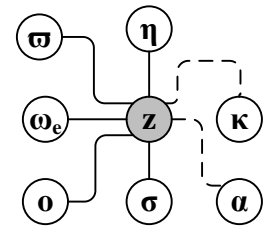

6)

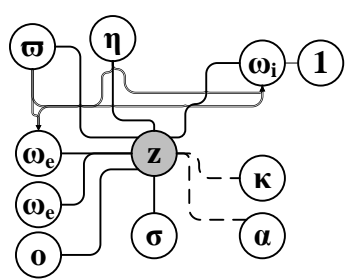

a Ventilation (IfcAirTerminal)
2)

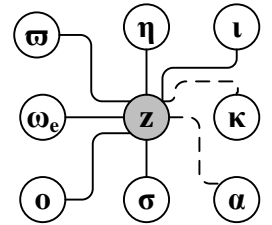

3)

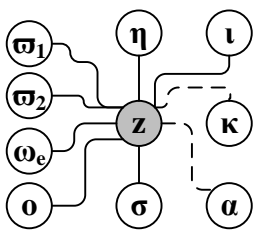

4)

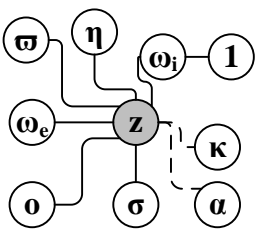

5)

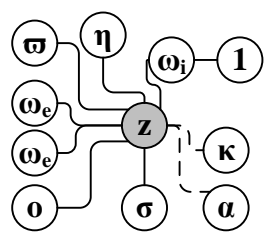

8)

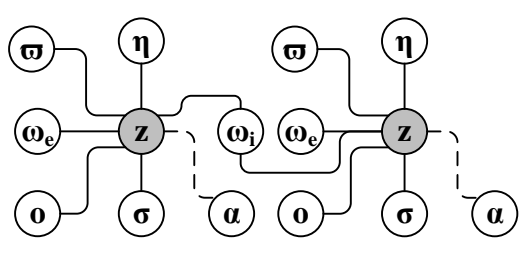
(๘) Windows (IfcWindow)
(z) Zone (IfcSpace-IfcZone)
(б) Sensors (IfcSensor)
External constructions
$\omega_{e}$ (IfcWallStandardCase, IfcSlab,
IfcRoof, IfcDoor)
Boundary constructions
(IfcWallStandardCase, IfcSlab,
IfcDoor)
(c) $\begin{aligned} & \text { Controller } \\ & \text { (IfcController) }\end{aligned}$
(o) Occupancy
(S) Slab (IfcSlab)
(1) Heaters (IfcSpaceHeater)
(a) Actuator (IfcActuator)

Figure 3: Eight topologies added to BII to generate different topologies compatible with grey box modelling

\subsection{Automated grey box model implementation}

As stated in section 5.1, the conversion of a topology into a Modelica model, occurs during the mapping process. The latter relies on a predefined association between nodes (e.g. the zone node $(z)$ in figure 3 ) and Modelica components referred to as Library template. For instance, table 3 presents the $\mathrm{Li}$ brary template used in this study. Each node presented in figure 3 is associated with a Modelica component in table 3. The equations embedded in the Modelica components, as well as required inputs, are also presented in table 3 .

The Library template is a flexible file that can be configured and modified by the users, i.e. the user can change the Modelica component corresponding to a specific node. For instance, a wall node $(\omega)$ can be represented by one resistance (1R) in a Library template (e.g. in table 4) or it can be represented by two resistances and one capacity (2R1C) (e.g. table 3). Similarly, the heater node $(\eta)$ can be represented by a simple gain component (see table 4) or an internal capacity and resistance (see table 3).

This flexibility in the definition of a Library template increases the possibility of adding complexity to the different topologies defined previously. Several Library templates defin- ing each node with different Modelica components can be used to vary the complexity of the generated model.

\section{Automated data acquisition for model training}

The toolchain generates a grey-box model structure for each topology, zone and Library template. This means that $\mathrm{n} * \mathrm{t} * \mathrm{~T}$ models are generated assuming that $\mathrm{n}$ is the number of zones and $t$ the number of topologies and $\mathrm{T}$ the number of library templates considered. Considering the number of structures that could be generated, it is difficult and very time consuming to link each model to the monitored data manually. For that reason, an automated measured data acquisition is key to reduce the manual configuration during the implementation.

Enriching BIM with real-time monitored sensor data (Dynamic-BIM) has recently been investigated to improve the use of BIM for facility management and operation. Such a combination provides the opportunity to visualise the different mechanical, electrical and plumbing components while having access to the real-time information. More importantly and in the present context, it allows to reduce the time required to retrieve measured data from a BMS. 
Table 3: Example of a library template providing the correspondence between Modelica components and nodes. Column 1: correspondence between node and entity. Column 2: Modelica component, column 3: function and input of the Modelica component, column 4: Description of the different connectors attached to the Modelica component.

\begin{tabular}{|c|c|c|c|}
\hline Nodes: Entity & Component & Modelica function & Connectors \\
\hline (Z): IfcSpace, IfcZone & $7 \pi$ & function: $C_{a i r} \frac{\mathrm{d} T_{a i r}}{\mathrm{~d} t}=\ldots$, input: $C_{a i r}=\# \mathrm{C \#}$. & HeatPort. \\
\hline $\begin{array}{l}\mathbf{w}_{\mathbf{e}} \text { and } \mathbf{w}_{\mathbf{i}} \text { (Mono-zone): } \\
\text { IfcWallStandardCase, } \\
\text { IfcSlab, IfcRoof }\end{array}$ & $\sum_{j}^{-1}$ & function: $C_{W} \frac{\mathrm{d} T_{W}}{\mathrm{~d} t}=\frac{T_{a i t}-T_{w e}}{\alpha_{1} * R}+\frac{T_{e}-T_{w e}}{\alpha_{2} * R}+\ldots$, input: $C_{W e}=\# \mathrm{C}, \mathrm{R}=\# \mathrm{R} \#$ & HeatPort, Input. \\
\hline $\begin{array}{l}\text { Wi }(\text { Multi-zone): } \\
\text { IfcSlab, If cDoor, } \\
\text { If cWallStandardCase }\end{array}$ & $\sum_{0}^{\frac{1}{I}}$ & function: $C_{W} \frac{\mathrm{d} T_{W}}{\mathrm{~d} t}=\frac{T_{a i t}-T_{w e}}{\alpha_{1} * R}+\frac{T_{e}-T_{w e}}{\alpha_{2} * R}+\ldots$, input: $C_{W e}=\# \mathrm{C} \#, \mathrm{R}=\# \mathrm{R} \#$ & HeatPort 1 , HeatPort 2. \\
\hline (1): Infiltration node & $\square=$ & function: $\frac{T_{a i t}-T_{\text {we }}}{R_{\text {inf }}}$, input: $R_{\text {inf }}: \# \operatorname{Rinf} \#$. & HeatPort, Input. \\
\hline $\begin{array}{l}\text { (1): If cWindow (with ori- } \\
\text { entation) }\end{array}$ & & $\begin{array}{l}\text { function: User-defined, it must include a component that compute the oriented solar } \\
\text { radiation, input:\#latitude\#, \#longitude\#, \#Azimuth\#, \#g\#, \#Area\#. }\end{array}$ & $\begin{array}{l}\text { Input, Input, Input, } \\
\text { HeatPort. }\end{array}$ \\
\hline $\begin{array}{l}\omega: \text { If cWindow (without } \\
\text { orientation) }\end{array}$ & 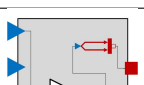 & function: $g * A *$ SolGloHor, input:\#g\#, \#Area\#. & $\begin{array}{l}\text { Input, Input, Input, } \\
\text { HeatPort. }\end{array}$ \\
\hline (k: Internal wall capacity & W. & function: User-defined. & bus 1, bus 2 . \\
\hline (7): IfcSpaceHeater & & function: $C \frac{\mathrm{d} T}{\mathrm{~d} t}=\frac{T_{a i t}-T}{\alpha_{1} * R}+Q_{H e a t}$, input:\#inc\#. & HeatPort. \\
\hline ( ): IfcAirTerminal & $\sqrt{\sqrt{10}}$ & function:... $+\dot{m} * \rho * C p\left(T_{S A}-T_{A i r}\right)+\ldots$, input:\#inc\#, \#azi\#, \#A\#, \#glazing\#. & $\begin{array}{l}\text { Input, } \quad \text { Input, } \\
\text { Input,HeatPort. }\end{array}$ \\
\hline (O): Occupant nodes & 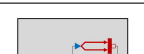 & function:... + Occ* OccGain + ..., input:\#inc\#, \#azi\#, \#A\#, \#glazing\#. & Input,HeatPort. \\
\hline 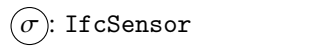 & $\triangle$ & function: Modelica.Thermal.HeatTransfer.Sensors.TemperatureSensor input: N/A. & Input,HeatPort. \\
\hline
\end{tabular}

Table 4: Example of a Library template in which wall nodes (w) and heater nodes $(\eta)$ are associated with simpler Modelica component compared to those in table 3

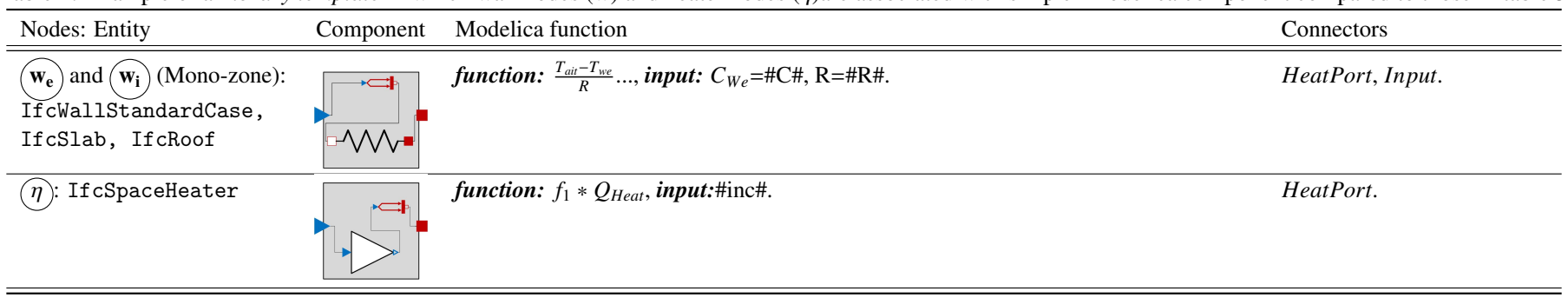

Several technical approaches have been proposed using various existing (proprietary or open) tools. For instance, Chiang et al. [54] developed a real-time replay system (RTRS) that combines the BIM software tool Autodesk Revit ${ }^{\mathrm{TM}}$ with the game engine Unity $3 d$ to display information from the sensors. Kensek [55] et al. connected light, humidity and $\mathrm{CO}_{2}$ sensors 
Table 5: Excerpt of a database table showing the columns corresponding to the outdoor temperature sensor and the room tenperature sensor defined in table 1

$\begin{array}{ccc}\text { Time stamp } & \text { RoomTemperature } & \text { OutdoorTemperature } \\ 2018-10-15 \text { 15:25 } & 15.5 & 22.1 \\ 2018-10-1515: 26 & 15.6 & 22.2 \\ 2018-10-1515: 27 & 15.7 & 22.3 \\ 2018-10-1515: 28 & 15.5 & 22.1 \\ \ldots & \ldots & \ldots\end{array}$

with BIM by combining Autodesk Revit ${ }^{\mathrm{TM}}$ API and the visual programming environment: Dynamo.

Such BIM and data integration requires a certain connection between BIM and physical sensors to uniquely tie a BIM object (e.g. a sensor object) with a specific physical sensor whose data is displayed. In that regard, the use of a common tag is generally used in the literature. For instance, Riaz et al. [56, 57] developed the Confined Space Monitoring System (CoSMoS) which combines wireless sensor devices, Autodesk Revit and a Structured Query Language server as a database management system. Revit's API is used to establish the connection between BIM and the database. The unique ID of the room (Room tag) in Revit is used to link the sensor located in the same room, allowing to tie the monitored data with a specific room. Similarly, Zhang et al. [58] propose the use of mark tag in IFC to act as a link between real-time data and BIM. A similar use of common labels or tags can also be found in Ref. [59, 60].

This study adopts a similar approach but instead relies on the Pset_DataBase (table 2) as the connection between BIM and BMS. The following points explain the implementation of the BIM-BMS connection:

\subsection{Construction stage: BMS configuration}

A Building Monitoring System (BMS) is a network of computerised devices that monitors both the environmental and indoor parameters of buildings to ensure the optimal control of the different integrated systems. It is assumed that the different sensing devices are installed according to the design BIM at the construction phase (figure 1, Construction phase). Specifically, the system integrator must carefully ensure that each sensor object (IfcSensor) defined in BIM is represented by the corresponding physical sensor.

Furthermore, it is assumed that the measured data is recorded in a centralised (relational) database such as a Structured Query Language (SQL) database in which the measured values are logged at a regular time step. Data from a specific physical sensor is specifically recorded in a column that bears an identifier set by the system integrator (e.g. table 5). Subsequently, the system integrator parametrises the Pset_DataBase property of the corresponding sensor object (IfcSensor). More specifically, the system integrator aligns the ColumnId parameter of the virtual sensor with the database column's name that stores the measured values from the corresponding physical sensor (i.e. ColumnId=RoomTemperature)

In this study, this connection between BIM and the database is implemented manually and is assumed to be performed by the system integrator at the construction phase. However, further study and research are still necessary to standardise and especially fully automate the implementation of this connection between BIM and BMS.

\subsection{Operational stage}

At the operational stage (see figure 1), an automated data acquisition routine is integrated into the toolchain. Its role is to retrieve the necessary data from the BMS and feed the different $\mathrm{RC}$ structures generated with measured data. In that regard, the automated data acquisition technique includes two components:

1. Firstly, a code routine parses the IFC file and identifies the sensor objects (IfcSensor) which are contained in the space considered. The temperature sensor objects included in the adjacent spaces, along with the outdoor temperature sensor and solar radiation sensor are identified as well. Subsequently, for each sensor or object previously identified, the code routine creates a Modelica input component (Modelica.Blocks.Interfaces.RealInput) at the top level of each grey-box Modelica model structure. Based on the object type or property or the IFC concept information between the sensor and the different building elements, these top-level Modelica inputs are connected to the RC components constituting the grey box model. For instance, a component representing an external wall is connected to the Modelica input component that represents the outdoor temperature sensor (IfcSensor with Pset_SensorTypeTemperatureSensor. TemperatureSensorType: OUTSIDETEMPERATURE ). Finally, the generated models are converted into Functional Mockup Units by using the PyFMI library [61]. This conversion allows to simulate the models inside a python-based environment and to take advantage of the existing optimisation and post-processing libraries available on this platform.

2. Secondly, another code routine retrieves the ColumnId parameters of the previously identified sensor objects and performs data queries to the SQL database. The retrieved data is then formatted and feed to the model through the Modelica input components.

From this point on, the different grey box models are connected to the BMS and optimisation algorithms can be used for the parameter estimation and model selection process.

\section{Model selection using multi-objective optimisation}

Any model selection method found in literature can be associated with the previously described grey-box model implementation approach. However, most optimisation algorithms used for the identification process include the interior point algorithm $[18,1]$ and the maximum likelihood estimation [20, 21, 22, 7] (using the CTSM-R package [44]). These algorithms require valid initial values which are calculated from the 
building characteristics. Furthermore, most of these algorithms use a single objective optimisation approach where the different parameters are identified based on a specific season of the year, resulting in a season specific model with limited capabilities for long-term simulations $[5,18]$.

This study investigates the use of the multi-objective evolutionary algorithm for the parameter estimation process. Genetic algorithms are rarely used in a parameter estimation approach since they sometimes find a near-optimum solution and are usually computationally expensive compared to conventional descent algorithm. Still, they can cope with a large solution space and they are more robust regarding the quality of the initial values [62, 63]. Among the studies that include genetic algorithms, and particularly the multi-objective optimisation algorithm NSGA-2, the approach proposed by Scanu et al. [63] presents two different objective functions to identify the tradeoff parameters between the thermal response from the aeraulic response. In the current study, the multi-objective genetic algorithm NSGA-2 serves to identify the impact of seasonally different data sets on the model accuracy and the long-term prediction performance. The focus is set on winter and summer datasets although autumn and spring data can be integrated as well.

A multi-objective optimisation finds the set of optimal solutions referred to as Pareto front. Each optimal solution or Pareto set consists of a set of model parameters (here RC parameters) that simultaneously satisfy the different objective functions. As an example, two objective functions are considered for each $\mathrm{RxCy}$ structure: the standardised Root Mean Square Error (sRMSE, equation 3) calculated during a winter-period ( $\left.s R M S E_{w}\right)$ and the sRMSE computed during a summer period ( $\left.s R M S E_{s}\right)$. Although period from swing seasons can be considered as well (by increasing the number of objective function), the goal here is to obtain a model that performs well during normal operation in summer as well as in winter. The selected Pareto set would be the one that presents a minimum difference between $s R M S E_{w}$ and $s R M S E_{s}$.

$$
s R M S E=\frac{1}{\max (\hat{T})-\min (\hat{T})} \sqrt{\frac{\sum_{i=1}^{n}\left(\hat{T}_{i}-T_{i}\right)^{2}}{n}}
$$

$\hat{T}_{i}$ and $T_{i}$ are the measured and simulated room temperature data points, $n$ the number of measured data points.

The multi-objective optimisation algorithm NSGA-2 from the Python package DEAP [64] is used. The initial values of the (RC) parameters are computed from equations 1 and 2.

The selection procedure compares the Pareto fronts resulting from the parameter estimation of the different structures $S t_{y}$. Subsequently, A cross-Validation is performed with the sRMSE as index. The structure that has a non dominated Pareto front combined with a low cross-validation SRMSE index is chosen as the optimal model among the structures generated.

\section{Case studies and experiment setup}

Although the diversity of grey box structures found in the literature is presented in section 1 , it is necessary to further demonstrate the relevance of the toolchain and show that the most appropriate RC structure is tied to the specific conditions and design of the building.

A fictive building (section 8.1) and an existing test facility (section 8.2) are considered to illustrate how the integration of BIM through BII can improve and facilitate the grey box modelling process. The focus is set on analysing lecture room type buildings. The characteristics of the case studies and the experiment conditions are detailed in the next section.

\subsection{Case 1: Fictive building}

This case study considers room 2 of the example case, presented in figure 2. From this BIM (figure 2) BII generates a detailed Modelica model using the IDEAS library [65]. The char-
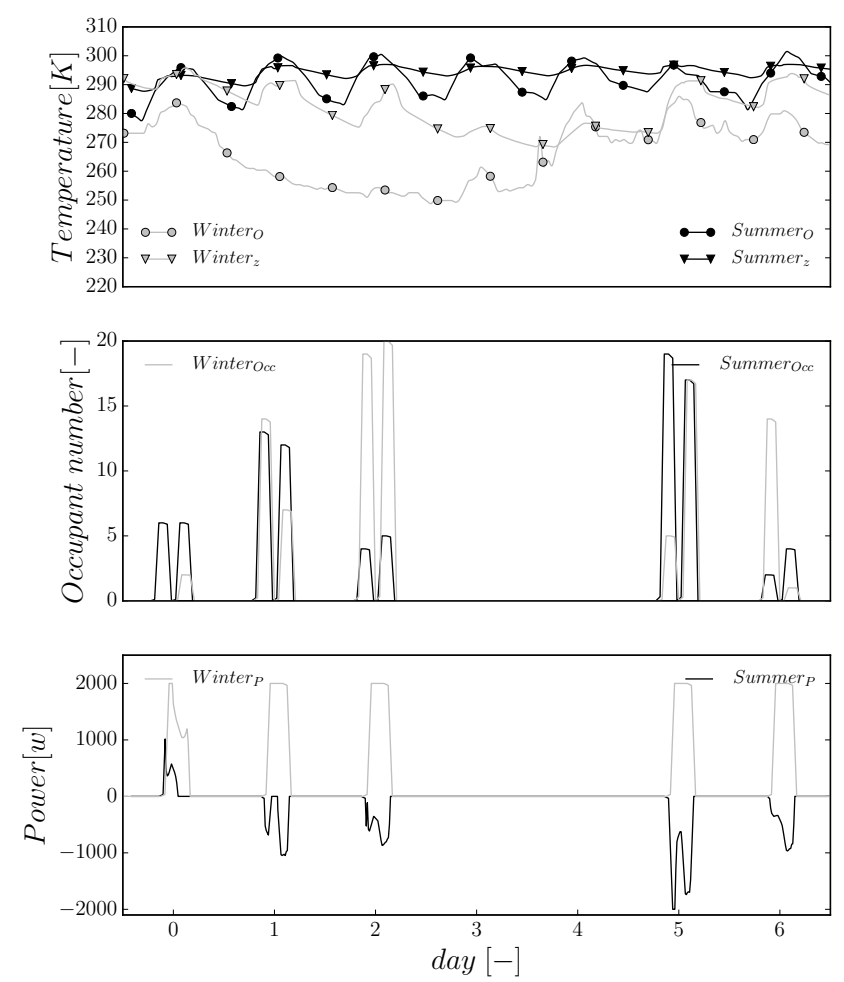

Figure 4: Profiles of the different input used for training. (Winter ${ }_{o}$ ): Outdoor temperature during the winter period, (Winter $)$ : Indoor temperature of the adjacent zone (1) during the winter period, $\left(\right.$ S ummer s $_{\text {) }}$ : Outdoor temperature during the summer period, (S ummer $)$ : Indoor temperature of the neighbouring

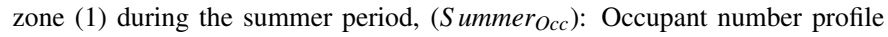
for the summer period considered, $\left(\right.$ Winter $\left._{O c c}\right)$ : Occupant number profile for the winter period considered, $\left(\right.$ S ummer $\left._{P}\right)$ : Power used for cooling during the summer period, $\left(\right.$ Winter $\left._{P}\right)$ : Power injected during the winter period

acteristics of the different construction elements (walls, roof, slab and windows) are taken from the BESTEST case 900 (see [53]). A dead-band controller activates the heating when the temperature is below $20^{\circ} \mathrm{C}$ while the zone is cooled when the temperature is above $24^{\circ} \mathrm{C}$. To simulate the schedule in a typical lecture room, the HVAC system is only activated during the working days and between 8 AM and 6 PM. In addition, a random number of occupants is attributed. The number of 
occupants in the morning (between 8 AM and 12 AM) differs from the number of occupants in the afternoon (1 PM to $6 \mathrm{PM}$ ). The standard BESTEST weather data file is used to generate the synthetic data.

Two seasonally different (winter and summer) data sets that represent typical operation conditions in lecture room type zones are synthesized. Figure 4 represents the different input profiles, i.e. the number of occupants (Winter ${ }_{O c c}$ and $S$ ummer $_{O c c}$ ), thermal power (Winter $P$ and $S$ ummer $_{P}$ ), outdoor temperature (Winter $O$ and Sumer $_{O}$ ) and indoor temperature of the neighbouring zone (Winter $Z$ and ummer $_{Z}$ ). These conditions are typical of a lecture room since these rooms are mostly unoccupied during the weekend and occupied with a variable number of occupants during the rest of the year.

\subsection{Case 2: Existing building}

The test facility is an existing university building located on the KU Leuven Technology Campus Ghent, Belgium (see figure 5). It comprises a staircase, a technical room and two lecture rooms (E120 and E220). The south-west facade windows are equipped with screens which are automatically closed when the solar radiation on that facade is higher than $250 \mathrm{~W} / \mathrm{m}^{2}$. Thermal comfort and indoor air quality in the lecture rooms are ensured by an air handling unit (figure 5.a) [42].

Figure 5.I gives a view of the facility's BIM with an emphasis on the two lecture rooms (E120 and E220). Figure 5.II provides a closer look on E220 which will be the focus of the current case study. It shows the air terminals (supply and returns) (figure 5.b), the temperature and $\mathrm{CO}_{2}$ sensors (figure 5.c) and the stereo camera (figure 5.d) placed above the entrance door of the room. The camera (figure 5.d) monitors the number of persons (in real-time) entering and leaving each lecture room. The number of occupants is stored in the building's monitoring system, more specifically into its SQL (Structured Query Language) database. Furthermore, this database contains information on the supply air temperature, the air volume flow, the actual indoor air temperature in both lecture rooms and the position of the screens and windows. The building is equipped with its own weather station which monitors the global solar irradiation and the outdoor temperature.

In the present case, seasonal dependence is checked based one week of winter data and one week of summer data. Figure 6 shows the different inputs for the considered winter and summer periods. It includes the outdoor temperature (Winter OutTemp $_{\text {, }}$

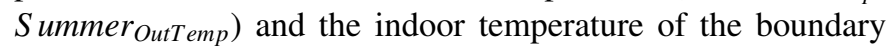
zones such as the staircase $\left(\right.$ Winter $_{T S T A I R}$, Summer $_{T S T A I R}$ ) and E120 (Winter WE120, Summer $_{T E 120}$ ). Also, the position of the screens (Winter E220SC $_{\text {S }}$ ummer $_{E 220 S C}$ ) and the number of occupants (Winter ${ }_{O c c E 220}$, Summer $_{O c c E 120}$ ) are presented. For both periods, the windows were kept closed. It can be deduced from figure 6 that the summer data depicts free float condition while normal operation data is used for the winter condition.

\section{Results and interpretation}

The single zone topologies (topology 1 to 6 in figure 3 ) coupled with the library template presented in table 3 are consid-

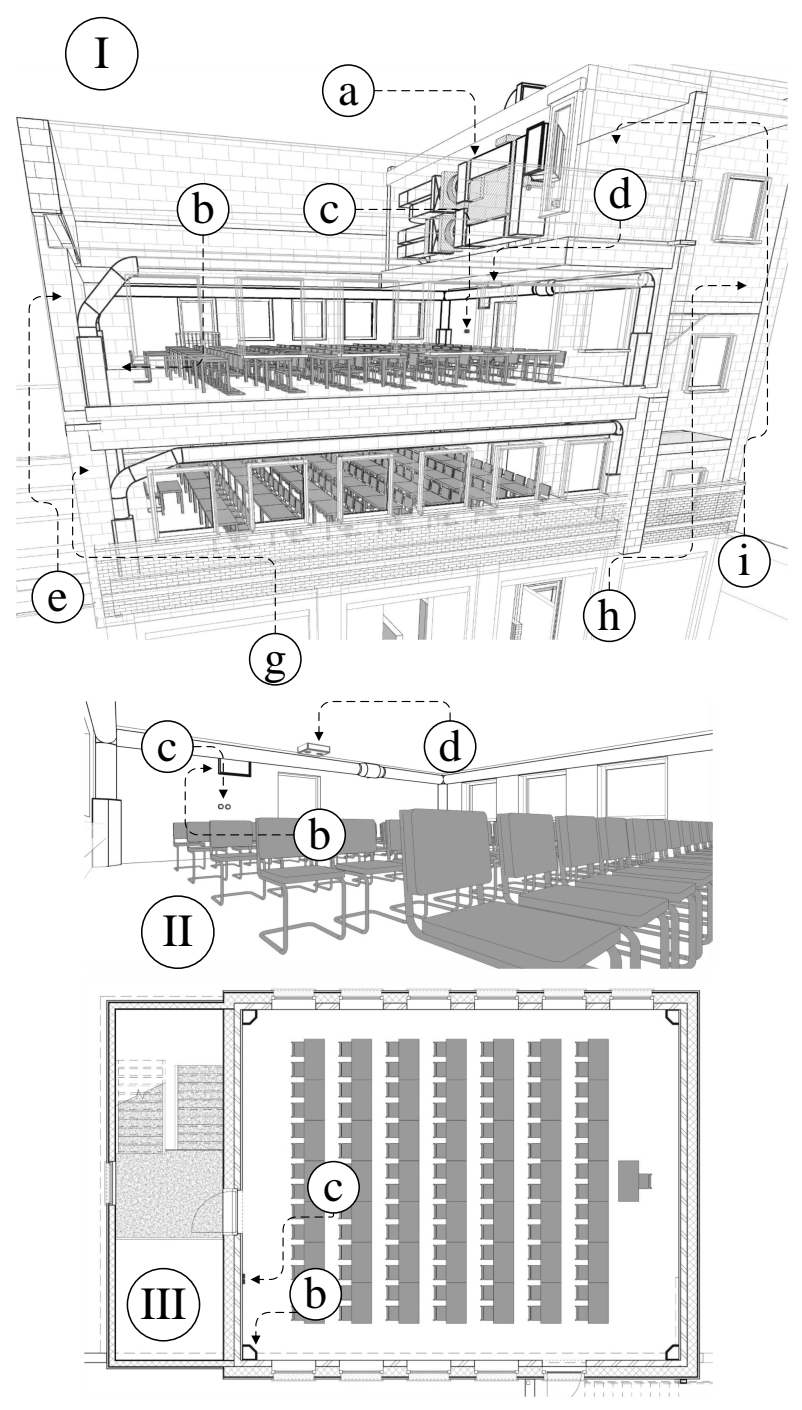

Figure 5: BIM of the test facility located on the KU Leuven Technology Campus Ghent, Belgium. (I): General 3D view of the case study. (II): Closer view of the inside of the lecture room E120. (III): Floor plan representing E120. (a): Air Handling Unit (AHU) located in the technical room. (b): (supply or return) air terminals. (c): sensors. (d): People counting stereo-camera. (e): room E220. (g): room E120. (h): staircase. (i): Technical room.

ered. More structures would be easily available by varying the library template.

Figure 7 shows the RxCy models generated from both case studies. The components represented by (Figure 7.1) are specific to case 1 while those represented by (Figure 7.2) are specific to case 2 .

These models are generated from the topologies 1 to 6 combined with the library template shown in table 3 . However, it is noteworthy to pinpoint that although the same topologies and library template were used, the resulting RxCy models differ depending on the building geometry and design. As an 

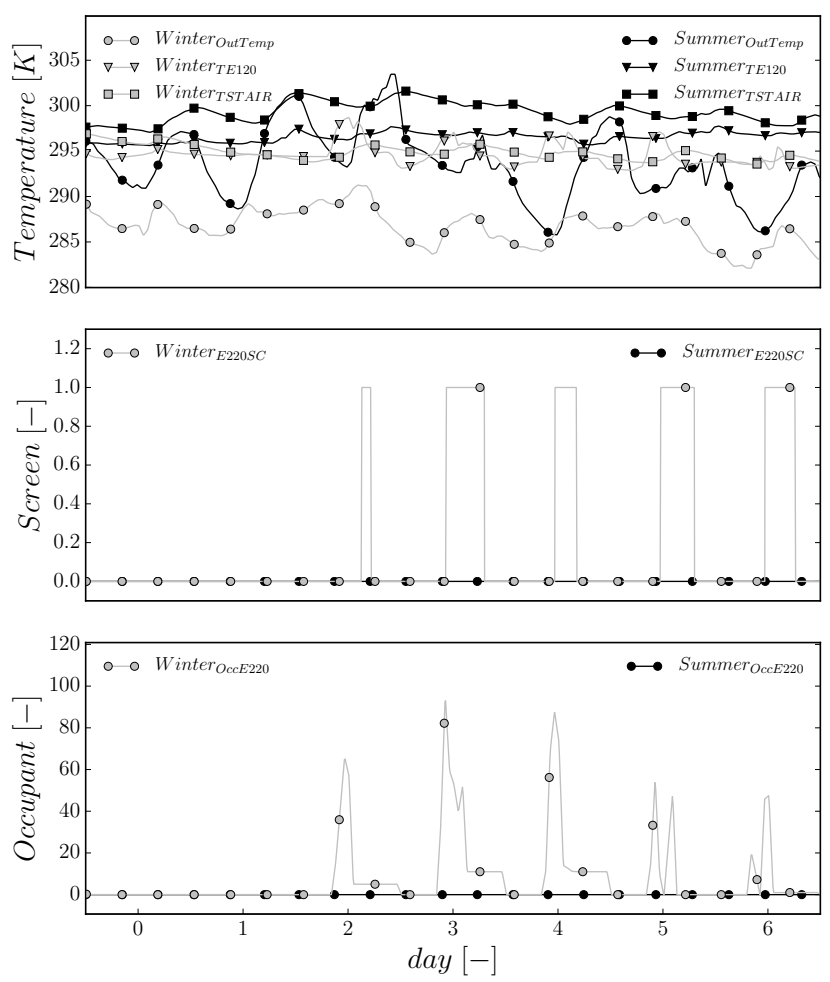

Figure 6: Presentation of the input sample used to train the different grey box structures. More specifically, the outdoor temperature and indoor temperature of the neighbouring zones, the positions of the screens (0: open, 1: Closed).

example, figure 7.St $t_{3}$ results from the use of topology 3 for both case studies. Since case 1 (figure 2) contains a heater (IfcSpaceHeater), the Modelica component associated with this device is instantiated in figure 7.S $t_{3}$. In contrast, case 2 does not include a heater but comprises a ventilation system. As a result, the ventilation $\left(Q_{v e n t}\right)$ is then instantiated.

The results obtained from the application of the selection process on both case studies are presented in the remainder of this section.

\subsection{Case 1: Fictive building}

Figure 8 shows the Pareto front of the different structures $\left(S t_{y}, \mathrm{y}=1\right.$ to 6$)$. The larger shapes represent the selected optimal Pareto set, i.e. the Pareto set that minimises the difference between $s R M S E_{s}$ and $s R M S E_{w}$. The closer this selected point is to $s R M S E_{s}=0$ and $s R M S E_{w}=0$, the better the model is.

From a general standpoint, it can be noted that more complex model structures $\left(S t_{3}\right.$ to $\left.S t_{6}\right)$ tend to result in a Pareto front closer to $s R M S E_{s}=0$ and $s R M S E_{w}=0$. The selected Pareto set (see section 7) of the less complex structure $\left(S t_{1}\right.$ see figure 7) has a standardised RMSE $s R M S E_{w}=0.068$ for the winter period and an $s R M S E_{s}=0.07$ for the summer period. Considering that the range $(\max (\hat{T})-\min (\hat{T}))$ is equal to $29.3 \mathrm{~K}$ in winter and $11.7 \mathrm{~K}$ in summer, the previous results indicate that $S t_{1}$ has an $R M S E_{s}=0.819 \mathrm{~K}$ and an $R M S E_{w}=1.9 \mathrm{~K}$. A slight improvement is noted by integrating the air infiltration model in $S t_{2}$ since the latter structure has an $s R M S E_{s}=0.069$ and an $s R M S E_{w}=0.067$ (see figure 8).

The structures $\left(S t_{3}, S t_{4}, S t_{5}\right.$ and $\left.S t_{6}\right)$ that include the orientations of the different windows (East and South, see figure 2) perform better than the two previous ones $\left(S t_{1}\right.$ and $\left.S t_{2}\right)$. For instance, the selected Pareto set of $S t_{3}$ has an $s R M S E_{s}=0.025$ and an $s R M S E_{w}=0.025$. $S t_{4}$ which is an extension of $S t_{3}$ that includes the boundary walls (see figure 7) has a lower performance than $S t_{3}$ with a selected Pareto set presenting an $s R M S E_{s}=0.028$ and an $s R M S E_{w}=0.028 . S t_{5}$, which is an extension of $S t_{4}$ and includes a separate modelling of the roof, is similar to $S t_{3}$ with an $s R M S E_{s}=0.024$ and an $s R M S E_{w}=0.024$. Finally, $S t_{6}$ includes the solar irradiation absorbed by the different construction elements and has an $s R M S E_{s}=0.026$ and an $s R M S E_{w}=0.026$.

Cross-Validation is performed to identify the structures that provide a good long-term prediction. Each structure $\left(S t_{y}, y=\right.$ 1 to 6) previously trained is simulated using three validation datasets with an increasing period. The first validation dataset $(1 w)$ spans over one week, the second $(2 w)$ spans over two weeks, and the last validation dataset is three weeks $(3 w)$ long.

Figure 9 shows the $s R M S E$ obtained by each structure $\left(S t_{y}\right)$ regarding the different validation datasets. Each row represents the results ( $s R M S E)$ obtained for each dataset $(w, 2 w, 3 w$, see top of figures). The two (2) right-side y-axes present the results obtained from the winter and summer period. The different structures $S t_{y}$ are ranked in an ascending order starting from the most accurate (i.e. minimum $s R M S E$ value) structure at the bottom to the least accurate one at the top. The left-side y-axis, on the other hand, corresponds to a ranking of the structures based on the average $s R M S E$ obtained for both seasons.

The validation confirms the results obtained in figure 8 since the structures $S t_{3}$ to $S t_{6}$ present a good prediction for the three periods considered. More specifically, $S t_{3}$ and $S t_{6}$ give the best validation results overall. For a one week prediction (figure 9), $S t_{6}$ has an $s R M S E_{s}=0.045$, an $s R M S E_{w}=0.037$ and an average $s R M S E$ equal to 0.041 while $S t_{3}$ has an $s R M S E_{s}=$ 0.052 , an $s R M S E_{w}=0.039$ and an average $s R M S E$ equal to 0.045 . These results show that the models are as effective in winter as in summer. A similar result is obtained for both two weeks and three weeks prediction ( $2 w$ and $3 w$ in figure 9). An average $s R M S E=0.034$ for $S t_{6}$ and an average $s R M S E=$ 0.033 for $S t_{3}$ are obtained for the two weeks prediction while $s R M S E=0.03$ for $S t_{6}$ and an average $s R M S E=0.034$ for $S t_{3}$ for the three weeks prediction.

Although the structures $\left(S t_{3}, S t_{4}, S t_{5}\right.$ and $\left.S t_{6}\right)$ have a similar performance, $S t_{3}$ is slightly more accurate for both seasons as shown in figure 8 . This shows that in this study, the added complexity in $S t_{4}$ to $S t_{6}$ has no added value to the accuracy of the model. This could be due to the fact that these structures have more parameters than $S t_{3}$ and might require more simulation runs to yield better results. A more elaborate dataset could also be used to improve the results from these structures.

As a result, for this case study, no definite conclusion can be drawn on the real impact of the increased complexity in $S t_{4}$, $S t_{5}$ and $S t_{6}$. However, it can be seen that the integration of the windows' orientation in $S t_{3}$ significantly impacts the accuracy 

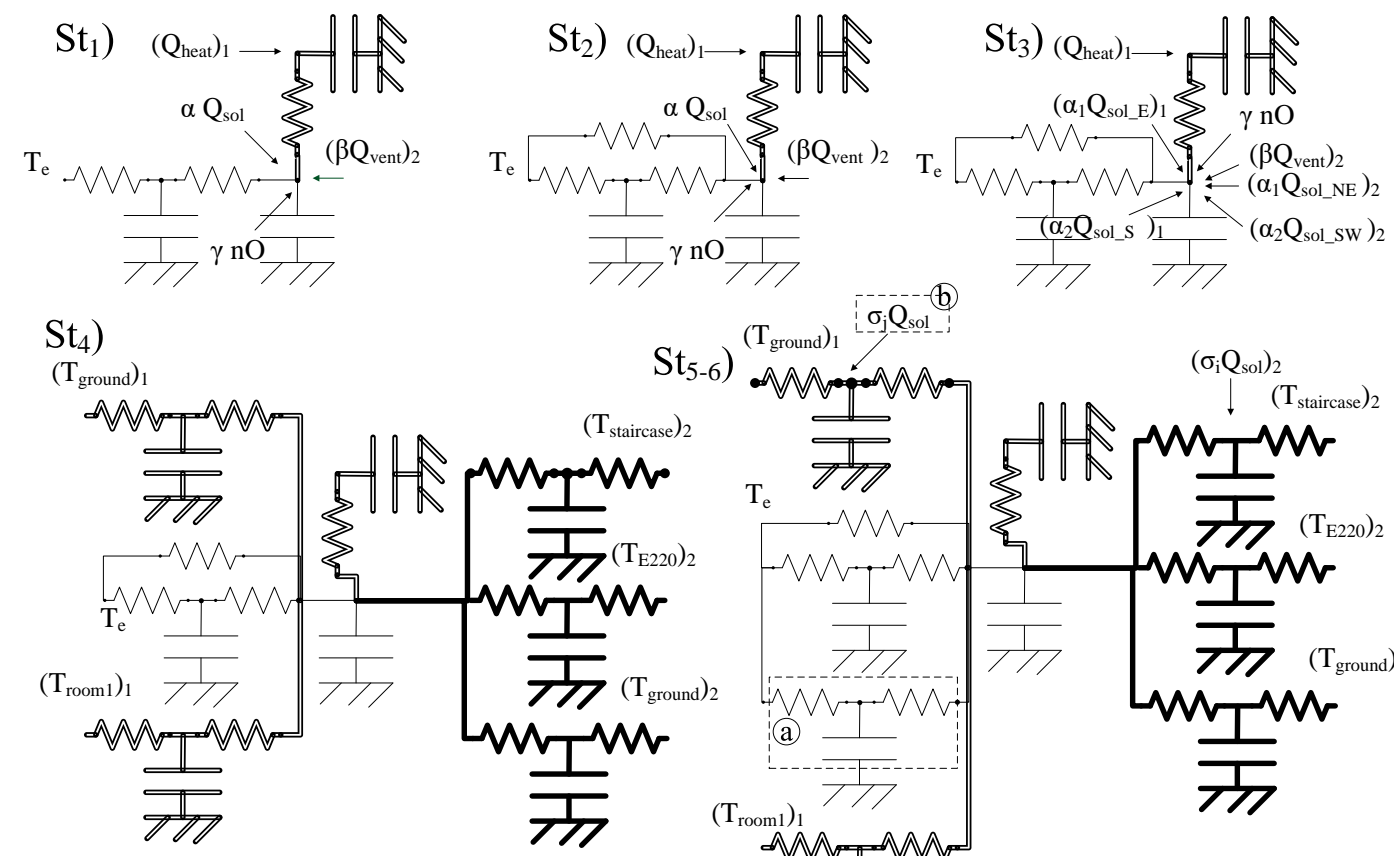
(1)

\section{(n)}

$\mathrm{T}_{\mathrm{e}}$

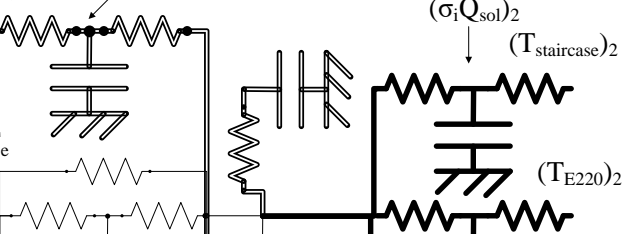

$\left(\sigma_{\mathrm{i}} \mathrm{Q}_{\mathrm{sol}}\right)_{2}$ $\left(\mathrm{T}_{\text {staircase }}\right)_{2}$

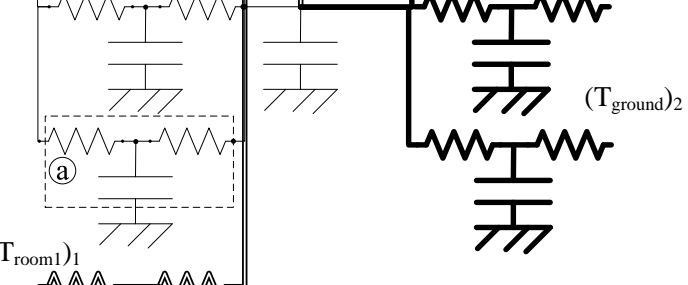

Common component for Case 1 and Case 2

1) $\begin{gathered}\text { Component specific for } \\ \text { Case } 1 .\end{gathered}$

2) $\mathcal{W}$ Component specific for Case 2.

Figure 7: Representation of the five model structures generated. $Q_{s o l}$ : Solar gains. $Q_{\text {sol }}$ : Solar gains following a direction. $Q_{v e n t}$ : Heat input from the ventilation. $Q_{\text {user }}$ : Heat input from occupant.

of the grey-box model.

Figures 10 and 11 show the comparison of the estimated parameters with theoretical values of the test case. More specifically, figure 10 compares the estimated UA-value of each structure $\left(S t_{y}\right)$ with the theoretical one (red line) computed from the values as reported in [53]. The estimated UA-value is computed from the inverse of the aggregated resistances between the indoor and outdoor temperature while its statistical distribution is derived from the different Pareto sets constituting the Pareto front. As presented in figure 10, the different structures $\left(S t_{y}\right)$ produce physically meaningful parameters which indicate that the structures are not overfitted [1]. This might result from the use of multi-objective optimisation with two seasonally different datasets. Also, the best structures $\left(S t_{3}\right.$ and $\left.S t_{6}\right)$ provide the best estimate of the theoretical UA-value showing that (1) the integration of the solar radiation with respect to windows orientation and (2) the inclusion of the radiative gains within the walls (see topology 6) can improve the accuracy of the parameter estimates. More importantly, such information is automatically integrated by using the here presented toolchain emphasising its relevance for grey box modelling.

Figure 11 compares the estimated and the theoretical thermal capacity. The estimated thermal capacity is computed from the sum of the different capacities in a model structure [7]. Its distribution is also derived from the Pareto sets constituting the Pareto front. Here, the theoretical thermal capacity (see red line) comprises the computed thermal capacity of the external and internal walls, roof and floor. As it can be seen in figure 11 , the estimated thermal capacities are lower than the theoretical ones. Such a result can be explained by the fact that only a part of the thermal mass is activated [21]. Nonetheless, the estimated capacities still have physical meaning and are relatively close to the theoretical one, confirming the validity of the previous result.

Finally, these results show that the most performant models $\left(S t_{3}\right.$ to $\left.S t_{6}\right)$ include the specific characteristics of the building advocating for the use of BIM to automatically generate the different model structures. $S t_{3}$ requires the orientations of the space's windows that can be automatically computed based on the information from BIM while $S t_{6}$ integrates BIM information related to the specific configuration of room 2 (e.g. Boundary walls). In a manual grey-box modelling approach, the information (e.g. windows orientation) needs to be manually retrieved per zone while in this study, the tool-chain retrieves such information automatically. Furthermore, the similarity of the results obtained for the three validation datasets demonstrates 


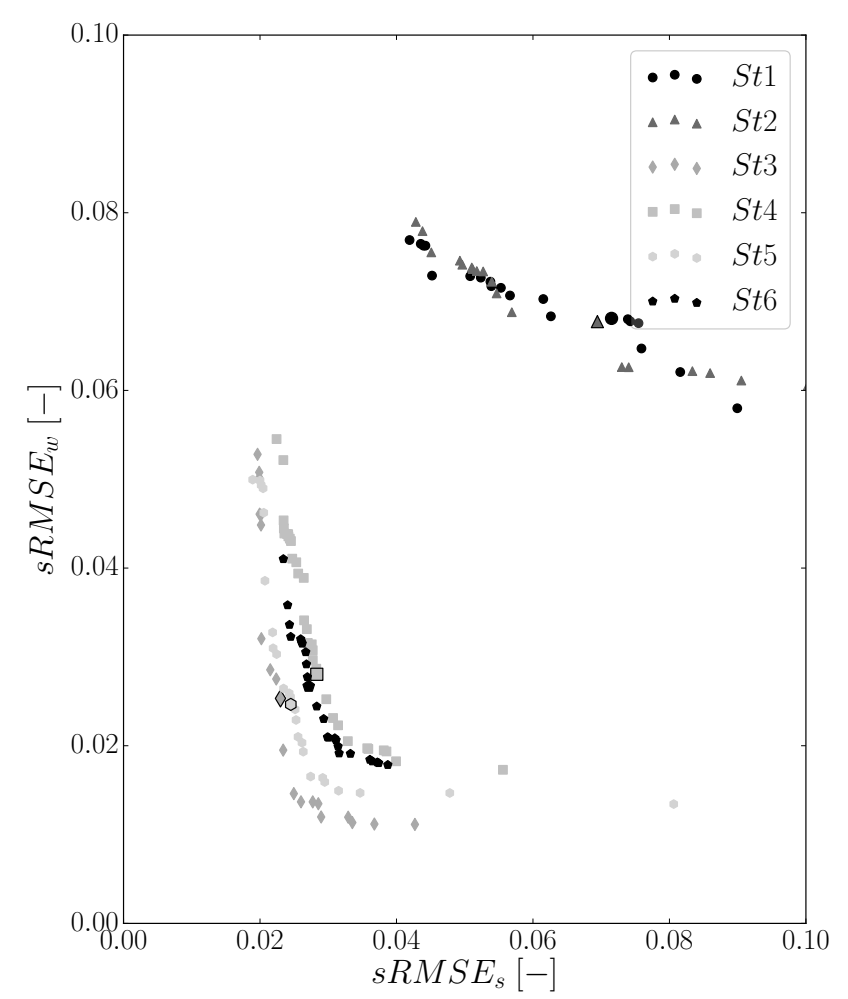

Figure 8: Comparison of the different Pareto sets obtained from the different structures $\left(S t_{1}\right.$ to $\left.S t_{6}\right)$

the robustness of the models with respect to the used prediction window and the season. Multi-objective optimisation such as NSGA-2 consequently provides grey box models that can produce good long-term results (here: up to three weeks open-loop simulation prediction) independently of the season.

\subsection{Case 2: Existing building}

This section presents the application of the toolchain to an existing building using real monitoring data. Similarly as in the previous case, the goal is to emphasize the importance of integrating the specific design characteristics of the building by using BIM information. In addition, the generated model is also used to detect a malfunctioning indoor temperature sensor.

Figure 7 shows the five structures used for the room E220. The components represented by figure 7.2 represent the RC components specific to the present case. These structures result from the same topologies and library template as used for the previous case. The differences come from the specific configuration of the two rooms (i.e. E220 and room 2 in figure 2).

From figure 12, it is shown that the structures $S t_{2}, S t_{3}$ and $S t_{5}$ have a Pareto front closer to $s R M S E_{s}=0$ and $s R M S E_{w}=0$. These three structures have a similar selected Pareto set equal to $s R M S E_{s}=0.08$ and $s R M S E_{w}=0.08$. However, the crossvalidation (for one week period) in figure 13 shows that more complex structures, especially $S t_{3}$, are the most accurate. For both seasons, $S t_{3}$ has a $R M S E_{s}=0.2$ and a $R M S E_{w}=0.2$. Also, the estimated UA-value for $S t_{3}$ has a mean of $158 \mathrm{~W} / \mathrm{K}$ with a standard deviation of $28 \mathrm{~W} / \mathrm{K}$. In comparison with the "theoretical value" $(123 \mathrm{~W} / \mathrm{K})$, a difference of $35 \mathrm{~W} / \mathrm{K}$ is noted. Nonetheless, the "theoretical value" does not include the potential impact of bad workmanship or thermal bridges. Consequently, no definite result can be drawn for such comparison. Regardless, the fact that the estimated value is physically meaningful indicates that the model is valid and not overfitted.

The achieved results confirm the dependence of the best model $\left(S_{3}\right)$ on the building's specific geometry and design (e.g. windows orientation) and thus emphasize the importance of the toolchain to automate the implementation of grey-box models.

In this specific case study, the integration of the heat exchange with adjacent zones through internal walls was not important, due to the separation by insulation layers, foreseen for test purposes. This heat exchange might be important for modelling in other buildings with normal, un-insulated internal walls and considerable temperature differences between adjacent zones.

\subsubsection{Small FDD example}

The resulting grey box model can be used for MPC or FDD. Figure 14 shows a simple example of these applications through the use of the resulting model for the detection of a manually introduced fault in an indoor temperature sensor. The temperature sensor errors have been induced in room E220 by blowing -with a hot air blower device - warm air towards the sensor. The experiment has been performed three times explaining the three peaks in figure 14. The measurement is compared to the model $\left(S t_{3}\right)$ prediction as shown in figure 14. The green area corresponds to the zone between simulation - RMS E and simulation + RMSE. RMSE being the Root Mean Square Error obtained from the cross-validation of $S t_{3}$ (see figure 13). A fault can be assumed if the measurement is outside the green area which is the case for the three induced peaks supposedly indicating errors during operation. Although it is a relatively trivial application, automating the implementation of grey box models enables the possibility to integrate such an FDD process automatically in buildings and ensure the perfect functioning of indoor sensors.

\section{Discussion}

On case study 1. The first case study (section 8.1) emphasises the relevance of the toolchain by demonstrating that models with increased complexity based on the building's specific design and geometry are potentially more accurate. As an example, the structure $\left(S t_{3}\right)$ that integrates the orientation of the windows is found the most accurate for both cases (case 1 and case 2). The generated models also depend on the building's specification given that $S t_{3}$ corresponds to a R4C3 in section 8.1while $S t_{3}$ is a R3C2 in section 8.2. Therefore, the use of a BIM to generate several structures enables the identification of the most appropriate for the considered case. In this study, only six RxCy structures were investigated although this number can be increased by combining the different topologies with 

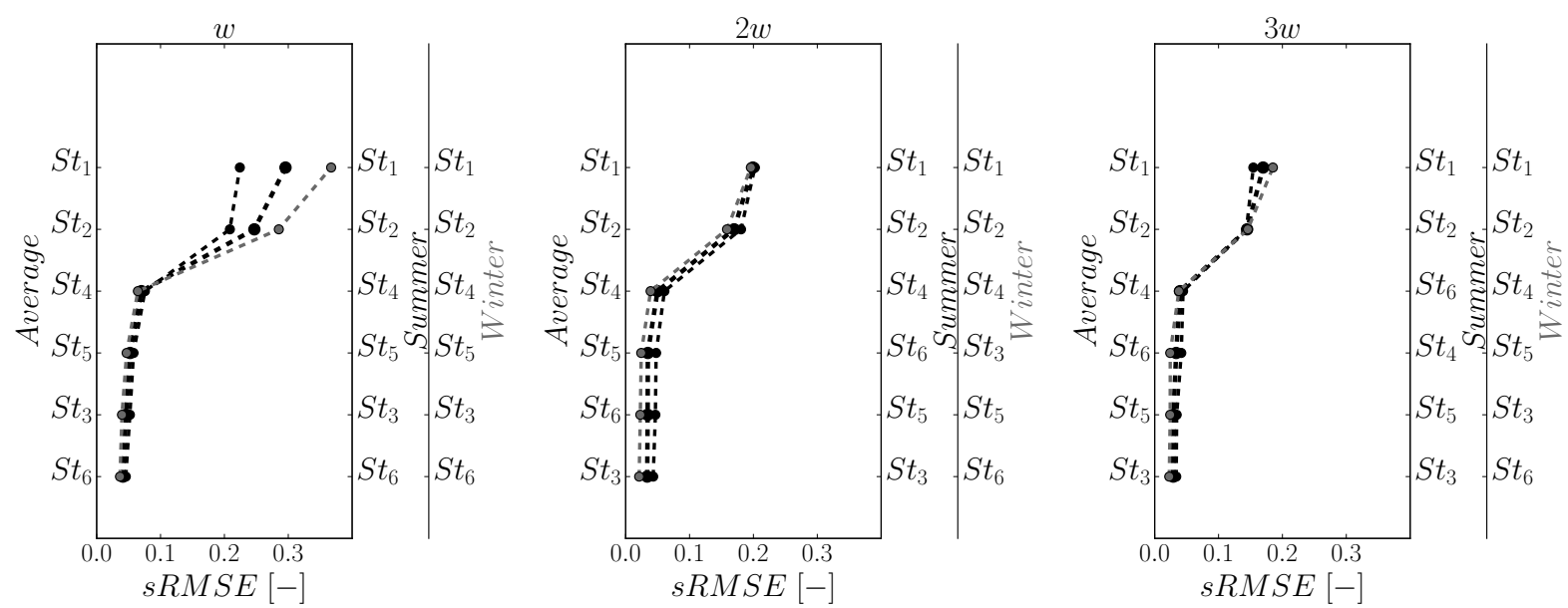

Figure 9: Comparison of the cross-validation sRMSE of the different structures trained using the different conditions. The validation data includes occupied summer condition (S ummer) and occupied winter condition (Winter). w: One week data (first column), $2 w$ : two-week data (second column), $3 w$ : three-week data (third column)

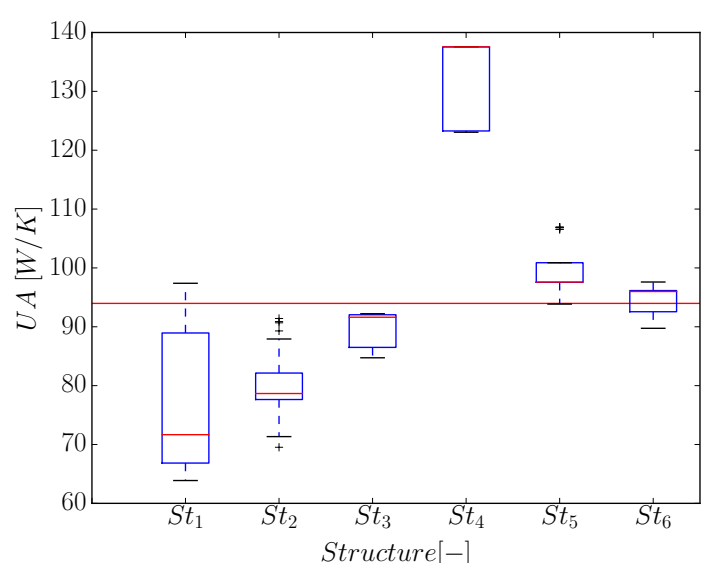

Figure 10: Estimated heat transfer coefficient: UA-value

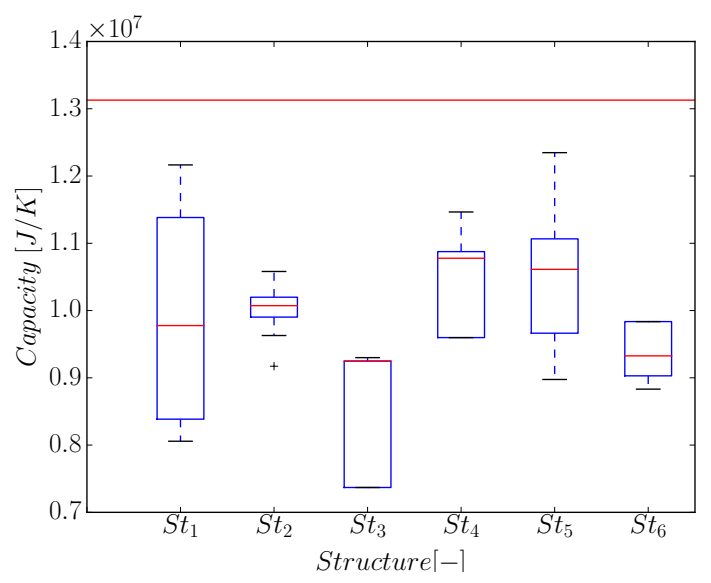

Figure 11: Estimation of the thermal capacity of the building envelope

different library templates in which the RC configuration of the

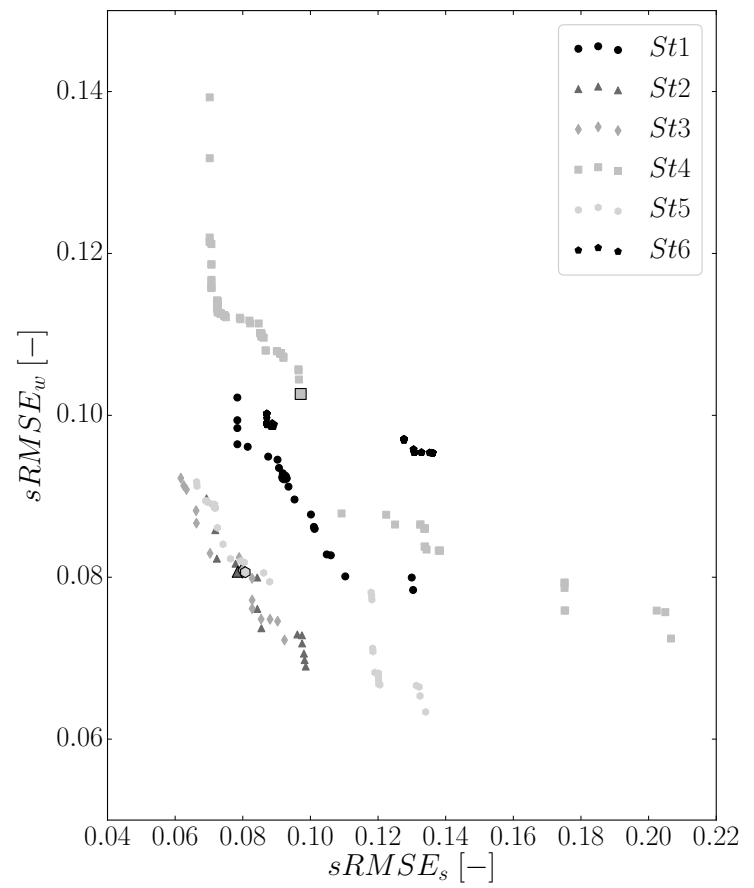

Figure 12: Comparison of the different Pareto front obtained from the different structures considered

different nodes are varied.

The use of a multi-objective optimisation ensures that the models are not limited to one season. Indeed, among the different input conditions tested, the combination of two seasonally different (winter and summer) datasets representing an occupied lecture room has generated models with satisfactory results (low sRMSE index $=0.03$ ) independently of the seasons and the occupancy conditions (occupied/unoccupied). 


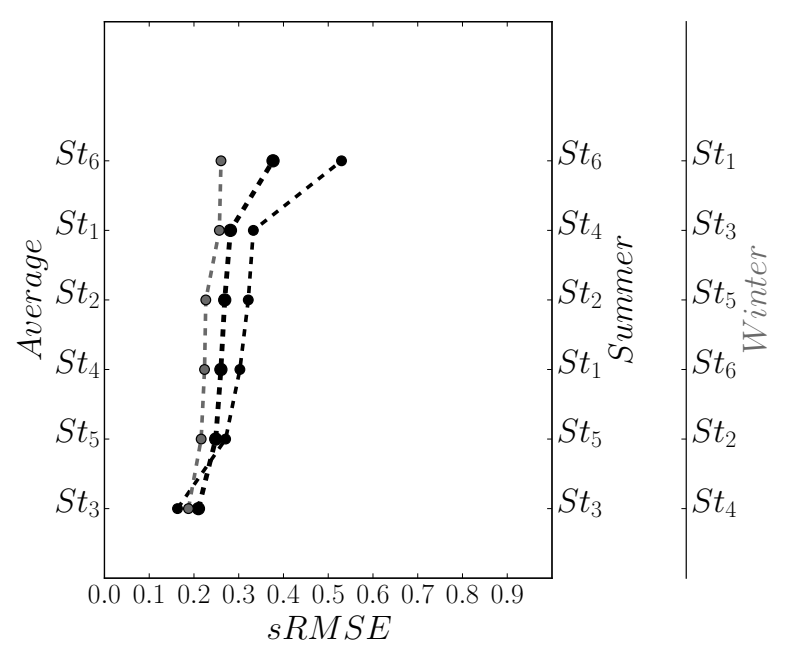

Figure 13: Cross-validation of the different structures

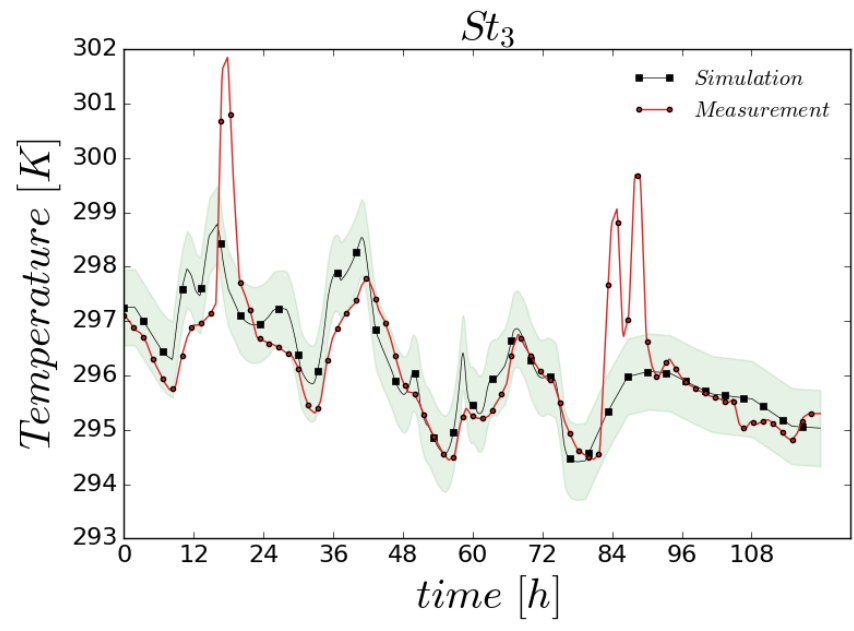

Figure 14: Application of the generated model for fault detection and diagnosis in indoor sensors

On case study 2. The application of the toolchain on case 2 demonstrates its use on a real building equipped with a monitoring system. The approach constitutes a powerful tool that can serve to generate automatically fast and accurate dynamic models of many types of buildings. The models are directly linked to the building monitoring system, thus minimising human involvement and hereby reducing the implementation cost and the risk of mistakes. The fact that the entire toolchain is embedded within a unique Python-based framework avoids switching between several tools. Although the toolchain is applied here to identify faults in indoor temperature sensors, it can be extended for more complex applications such as Model Predictive Control (MPC). The use of the toolchain therefore provides an interesting opportunity to facilitate the implementation of these type of strategies in buildings.
General. The model generation process (section 5) can be combined with any other forward selection procedure (e.g. [1]) but the choice of a multiobjective optimisation approach is motivated by its robustness towards low-quality initial values to facilitate its use with a BIM generated from 3D reconstruction processes. Multiobjective optimisations also provide models independent of seasonal influences, as presented in section 8.1. However, further research is needed to reduce the computational cost and several thousand simulation evaluations (here: 24000) are required to obtain models with acceptable accuracy. Considering that the simulation of an FMU can last from 1 to several seconds depending on the complexity of the model's structure, the training period varies from several hours to several days. In addition, evolutionary algorithms are known to find near-optimum solutions. Therefore, fast local optimisers (e.g. IPOPT) could be used to refine the results obtained from the NSGA-2, if required. In addition, the use of seasonally different datasets means that multiobjective optimisation as used in this study cannot be used directly after building completion. This stresses the importance of the flexibility of the presented toolchain. For instance, at the building completion, the automated grey box generation approach is coupled with the forward selection presented in [1], while at a later stage multiobjective optimisation could be used, to obtain data likely to produce accurate long-term prediction.

Furthermore, the approach potentially reduces the risk of errors, by automatically identifying the model inputs. As an example, air terminals are present in the case 2 which means that a ventilation component needs to be instantiated. Similarly, the definition of an IfcSpaceHeater leads to the implementation of a heater component.

\section{Conclusion}

Several energy-saving strategies including Model Predictive Control (MPC) require fast and accurate dynamic models of the building. Electrical analogy (RC) models or grey-box models satisfy these requirements and are widely used in literature. However, the identification of the optimal RC-structure represents a significant cost. A conventional approach consists of manually implementing several model (RC) structures with an increasing level of complexity and then performing a forward selection procedure to identify the most suitable.

This study aims at facilitating grey box implementation procedure by using information from BIM to generate different model ( $\mathrm{RxCy}$ ) structures that reflect the considered building's specific geometry and design. Furthermore, the use of a multiobjective optimisation procedure for the selection process is investigated. The model parameters are specifically estimated by the simultaneous resolution of two objective functions computed from two different data sets (winter and summer).

An earlier developed IFC to Modelica translation approach referred to as BII has been adapted in this study to generate several RxCy structures with different levels of complexity. BII has been modified to create additional topologies ranging from simple to more complex representations. These additional topologies combined with the possibility to use different library tem- 
plates increase the number of structures that can be generated per topology and consequently the number of possible $\mathrm{RxCy}$ structures. Also, information from the BIM is used to compute initial RC-values.

The approach has been applied to two case studies. The first is a fictive building based on the properties of the BESTEST case 900, while the second is an existing university building located on the KU Leuven Technology Campus Ghent. The multi-objective optimisation algorithm NSGA-2 has been applied during the forward selection process. Two objective functions have been evaluated during a winter period and a summer period for each model structure for the parameter estimation.

This study underlines the high relevance of the developed toolchain for grey box modelling. The integration of the specific characteristics of a building such as windows orientation has been shown to produce grey box models with a better accuracy (i.e. lower sRMSE index). The results of the case studies demonstrate that using specific design information of a building can improve the accuracy of the generated model. Consequently, the inclusion of BIM for grey box modelling not only allows to automate grey box implementation, but also has the potential to yield better models.

Further development of the hereby presented automated approach can focus on the following points:

- As stated in section 6, further work is necessary to establish an automated strategy to couple virtual sensors in BIM and the corresponding physical sensors.

- The present study focuses on automatically implementing a lumped RC model of the building's outer shell from BIM. Future development could consider a combined implementation of RC models with simplified building system models from BIM. The approach presented in Ref [42] can serve as a starting point.

- This research uses a multi-objective optimisation algorithm for the model selection method. A comparison of the advantages of multi-objective optimisation and conventional single objective optimisation approaches for grey box modelling can prove useful and is part of future studies.

- Finally, the coupling of the tool-chain with more elaborate FDD and MPC schemes is a next step for the development of the tool-chain.

\section{Aknowledgement}

This project receives the support of the European Union, the European Regional Development Fund ERDF, Flanders Innovation \& Entrepreneurship and the Province of Limburg and internal funding of KU Leuven.

\section{References}

[1] R. D. Coninck, F. Magnusson, J. Åkesson, L. Helsen, Toolbox for development and validation of grey- box building models for forecasting and control, Journal of Building Performance Simulation 9 (3) (2016) 288303. doi : 10.1080/19401493.2015.1046933.
[2] M. Avci, M. Erkoc, A. Rahmani, S. Asfour, Model predictive HVAC load control in buildings using real-time electricity pricing, Energy \& Buildings 60 (2013) 199-209. doi:10.1016/j.enbuild.2013.01.008.

[3] T. H. Pedersen, R. E. Hedegaard, S. Petersen, Space heating demand response potential of retrofitted residential apartment blocks, Energy \& Buildings 141 (2017) 158-166. doi:10.1016/j.enbuild.2017.02. 035.

[4] F. Jorissen, Toolchain for Optimal Control and Design of Energy Systems in Buildings, Ph.D. thesis, KU Leuven (2018) [cited 2018-08-14] URL https://lirias.kuleuven.be/1652305

[5] F. Ferracuti, A. Fonti, L. Ciabattoni, S. Pizzuti, A. Arteconi, L. Helsen, G. Comodi, Data-driven models for short-term thermal behaviour prediction in real buildings, Applied Energy 204 (2017) 1375-1387. doi : 10.1016/j . apenergy. 2017.05.015.

[6] R. E. Hedegaard, S. Petersen, Evaluation of Grey-Box Model Parameter Estimates Intended for The Thermal Characterization of Buildings, Energy Procedia 132 (2017) 982-987. doi:10.1016/j.egypro.2017. 09.692.

[7] P. D. Andersen, H. Madsen, C. Rode, Characterization of heat dynamics of an arctic low-energy house with floor heating, Building simulation 7 (6) (2014) 595-614. doi : 10.1007/s12273-014-0185-4.

[8] C. van Dronkelaar, M. Dowson, E. Burman, C. Spataru, D. Mumovic, A Review of the energy Performance Gap and its Underlying Causes in Non-Domestic Buildings, Frontiers in Mechanical Engineering 1 (17). doi: 10.3389/fmech.2015.00017.

[9] P. D. Wilde, The gap between predicted and measured energy performance of buildings : A framework for investigation, Automation in Construction 41 (2014) 40-49. doi: 10.1016/j. autcon.2014.02.009.

[10] S. Niu, W. Pan, Y. Zhao, A virtual reality integrated design approach to improving occupancy information integrity for closing the building energy performance gap, Sustainable Cities and Society 27 (2016) 275-286. doi:10.1016/j.scs.2016.03.010.

[11] M. Herrando, D. Cambra, M. Navarro, L. De, G. Millán, I. Zabalza, Energy Performance Certification of Faculty Buildings in Spain : The gap between estimated and real energy consumption, Energy Conversion and Management 125 (2016) 141-153. doi:10.1016/j .enconman. 2016. 04.037.

[12] X. Xu, S. Wang, A simplified dynamic model for existing buildings using CTF and thermal network models, International Journal of Thermal Sciences 47 (2008) 1249-1262. doi:10.1016/j.ijthermalsci. 2007. 10.011.

[13] G. Mustafaraj, J. Chen, G. Lowry, Thermal behaviour prediction utilizing artificial neural networks for an open office, Applied Mathematical Modelling 34 (11) (2010) 3216-3230. doi : 10.1016/j . apm . 2010.02.014.

[14] A. Ashtiani, P. A. Mirzaei, F. Haghighat, Indoor thermal condition in urban heat island : Comparison of the artificial neural network and regression methods prediction, Energy \& Buildings 76 (2014) 597-604. doi : 10.1016/j.enbuild.2014.03.018. URL http://dx.doi.org/10.1016/j.enbuild.2014.03.018

[15] G. Mustafaraj, J. Chen, G. Lowry, Development of room temperature and relative humidity linear parametric models for an open office using BMS data, Energy and Buildings 42 (2010) 348-356. doi : 10.1016/j . enbuild.2009.10.001.

[16] J. C.-m. Yiu, S. Wang, Multiple ARMAX modeling scheme for forecasting air conditioning system performance, Energy Conversion and Management 48 (2007) 2276-2285. doi : 10.1016/j. enconman . 2007.03. 018.

[17] M. Killian, M. Kozek, Ten questions concerning model predictive control for energy ef fi cient buildings, Building and Environment 105 (2016) 403-412. doi: 10.1016/j.buildenv.2016.05.034.

[18] T. Berthou, P. Stabat, R. Salvazet, D. Marchio, Development and validation of a gray box model to predict thermal behavior of occupied office buildings, Energy \& Buildings 74 (2014) 91-100. doi : 10.1016/j. enbuild.2014.01.038.

[19] V. S. K. V. Harish, A. Kumar, Reduced order modeling and parameter identification of a building energy system model through an optimization routine, Applied Energy 162 (2016) 1010-1023. doi : 10.1016/j. apenergy . 2015.10.137.

[20] J. Terés-zubiaga, C. Escudero, C. García-gafaro, J. M. Sala, Methodology for evaluating the energy renovation effects on the thermal performance of social housing buildings : Monitoring study and grey box 
model development, Energy \& Buildings 102 (2015) 390-405. doi: 10.1016/j.enbuild.2015.06.010.

[21] G. Reynders, J. Diriken, D. Saelens, Quality of grey-box models and identified parameters as function of the accuracy of input and observation signals, Energy \& Buildings 82 (2014) 263-274. doi : 10.1016/j . enbuild.2014.07.025

[22] P. Bacher, H. Madsen, Identifying suitable models for the heat dynamics of buildings, Energy \& Buildings 43 (2011) 1511-1522. doi : 10.1016/ j.enbuild.2011.02.005.

[23] D. Ilter, E. Ergen, BIM for building refurbishment and maintenance : current status and research directions, Structural Survey 33 (3) (2015) 228256. doi:10.1108/SS-02-2015-0008

[24] Y. Liu, S. V. Nederveen, M. Hertogh, Understanding effects of BIM on collaborative design and construction : An empirical study in China, International Journal of Project Management 35 (2017) 686-698. doi : 10.1016/j.ijproman.2016.06.007.

[25] A. Ghaarianhoseini, J. Tookey, A. Ghaarianhoseini, N. Naismith, S. Azhar, O. Emova, K. Raahemifar, Building Information Modelling ( BIM ) uptake : Clear benefits, understanding its implementation, risks and challenges, Renewable and Sustainable Energy Reviews 75 (September 2016) (2017) 1046-1053. doi:10.1016/j.rser.2016.11.083.

[26] H.-y. Chong, C.-y. Lee, X. Wang, A mixed review of the adoption of Building Information Modelling ( BIM ) for sustainability, Journal of Cleaner Production 142 (2017) 4114-4126. doi : 10.1016/j . jclepro . 2016.09.222.

[27] W. Jeong, J. B. Kim, M. J. Clayton, J. S. Haberl, W. Yan, Translating Building Information Modeling to Building Energy Modeling Using Model View Definition, The Scientific World Journal (2014) 121doi:10.1155/2014/638276.

[28] J. B. Kim, W. Jeong, M. J. Clayton, J. S. Haberl, W. Yan, Developing a physical BIM library for building thermal energy simulation, Automation in Construction 50 (2014) 16-28. doi:10.1016/j.autcon.2014.10. 011.

[29] V. Dimitriou, S. Firth, T. Hassan, F. Fouchal, BIM enabled building energy modelling : development and verification of a GBXML to IDF conversion method, in: Proceedings of the 3rd IBPSA-England Conference BSO 2016, Great North Museum, Newcastle, 12th-14th September 2016. no. 1126,2016 URL http: //www.ibpsa.org/proceedings/BS02016/p1126.pdf

[30] C. Wang, Y. K. Cho, Automated gbXML-Based Building Model Creation for Thermal Building Simulation, in: IEEE Xplore and CSDL, The International Conference on 3D Vision, Univ. of Tokyo, Tokyo, Japan, Dec. 8-11, 2014, 2014. doi: 10.1109/3DV. 2014.109.

[31] K.-u. Ahn, Y.-j. Kim, C.-s. Park, I. Kim, K. Lee, BIM interface for full vs . semi-automated building energy simulation, Energy \& Buildings 68 (2014) 671-678. doi : 10.1016/j.enbuild.2013.08.063.

[32] I. J. Ramaji, J. I. Messner, R. M. Leicht, Leveraging Building Information Models in IFC to perform energy analysis in Openstudio, in: ASHRAE and IBPSA-USA SimBuild 2016, 2016.

URL http://ibpsa-usa.org/index.php/ibpusa/article/ view/365

[33] A. Cemesova, C. J. Hopfe, R. S. Mcleod, PassivBIM : Enhancing interoperability between BIM and low energy design software, Automation in Construction 57 (2015) 17-32. doi:10.1016/j . autcon.2015.04. 014.

[34] A. Cormier, S. Robert, P. Roger, L. Stephan, E. Wurtz, Towards a BIMbased service oriented platform: Application to building energy performance simulation, in: Proceedings of Building Simulation 2011: 12th Conference of International Building Performance Simulation Association, Sydney, 14-16 November., 2011, pp. 14-16.

[35] H. Kim, Z. Shen, I. Kim, K. Kim, A. Stumpf, J. Yu, BIM IFC information mapping to building energy analysis (BEA ) model with manually extended material information, Automation in Construction 68 (2016) 183193. doi : 10.1016/j . autcon.2016.04.002.

[36] X. Yang, S. Ergan, Towards a Formal Approach for Determining Functions of HVAC Components Represented in IFC, in: International Conference on Computing in Civil and Building Engineering, 2014, pp. 633640.

[37] S. Robert, D. Mazza, B. Hilaire, P. Sette, B. Vinot, An approach to enhancing the connection between BIM models and building energy simulation HVAC systems in the loop, in: 10th European Conference on
Product and Process Modelling, 2014. doi:10.1201/b17396-28.

[38] M. Wetter, Modelica-based modelling and simulation to support research and development in building energy and control systems, Journal of Building Performance Simulation 2 (june) (2009) 143-161. doi : 10.1080/19401490902818259.

[39] J. L. M. Hensen, R. Lamberts, Building performance simulation for design and operation, Spon Press, 2011.

[40] P. Remmen, J. Cao, S. Ebertshäuser, J. Frisch, M. Lauster, T. Maile, D. Müller, C. V. Treeck, An open framework for integrated BIM-based building performance simulation using Modelica, in: 14th International Conference of the International Building Performance Simulation Association (IBPSA), BS2015, no. 2384, 2015, pp. 1-8.

URL http: //www.ibpsa.org/proceedings/BS2015/p2384.pdf

[41] J. O’Donnell, R. See, C. Rose, T. Maile, V. Bazjanac, P. Haves, SIMMODEL: A domain data model for whole building energy simulation, in: Proceedings of Building Simulation 2011: 12th Conference of International Building Performance Simulation Association, Sydney, 14-16 November., 2011, pp. 1-8.

URL http://www.ibpsa.org/proceedings/BS2011/P\{_\}1223. pdf

[42] A. Andriamamonjy, D. Saelens, R. Klein, An automated IFC-based workflow for building energy performance simulation with Modelica, Automation in Construction 91 (2018) 166-181. doi:10.1016/j.autcon. 2018.03 .019$.

[43] S. Wang, X. Xu, Simplified building model for transient thermal performance estimation using GA-based parameter identification, International Journal of Thermal Sciences 45 (2006) 419-432. doi:10.1016/ j.ijthermalsci.2005.06.009.

[44] R. Juhl, J. K. Møller, H. Madsen, ctsmr Continuous Time Stochastic Modeling in R, The R Journal (2015) 1-11arXiv:arXiv:1606. $00242 \mathrm{v} 1$.

[45] Z. Pezeshki, S. Ali, S. Ivari, Applications of BIM : A Brief Review and Future Outline, Archives of Computational Methods in Engineering 25 (2018) 273-312. doi : 10.1007/s11831-016-9204-1.

[46] SmartMarket Report, The business value of BIM in China, 2015.

[47] SmartMarket Report, The business value of BIM in North America: Multi-year trend analysis and user ratings (2007-2012), 2012.

[48] National Building Specification, National BIM Report 2017, Tech. rep. (2017) [cited 2018-08-14]

URL nbs-national-bim-report-2017

[49] Smartmarket Report, The Business Value of BIM in Europe, 2010.

[50] J. Široky, F. Oldewurtel, J. Cigler, S. Prívara, Experimental analysis of model predictive control for an energy efficient building heating system, Applied Energy 88 (2011) 3079-3087. doi:10.1016/j .apenergy . 2011.03 .009$.

[51] Y. Zong, G. Martin, R. Mirra, S. You, J. Hu, X. Han, Challenges of implementing economic model predictive control strategy for buildings interacting with smart energy systems, Applied Thermal Engineering 114 (2017) 1476-1486. doi:10.1016/j.applthermaleng.2016.11.141.

URL http://dx.doi.org/10.1016/j.applthermaleng. 2016 . 11.141

[52] T. Liebich, T. Chipman, Industry Foundation Classes, Version 4 - Addendum 1 (2015).

[53] R. Judkoff, J. Neymark, International Energy Agency Building Energy Simulation Test (bestest) and Diagnostic Method., Tech. rep., National Renewable Energy Lab.,, Golden, CO. (1995) [cited 2018-08-14]. URL https ://www.nrel.gov/docs/legosti/old/6231.pdf

[54] C.-t. Chiang, C.-p. Chu, C.-c. Chou, BIM-enabled power consumption data management platform for rendering and analysis of energy usage patterns, Procedia Engineering 118 (2015) 554-562. doi:10.1016/j . proeng. 2015.08.480. URL http://dx.doi .org/10.1016/j.proeng. 2015.08.480

[55] K. M. Kensek, Integration of Environmental Sensors with BIM : case studies using Arduino, Dynamo, and the Revit API, Informes de la Construcción 66. doi:10.3989/ic.13.151.

[56] Z. Riaz, M. Arslan, A. K. Kiani, S. Azhar, Automation in Construction CoSMoS : A BIM and wireless sensor based integrated solution for worker safety in confined spaces, Automation in Construction 45 (2014) 96-106. doi:10.1016/j.autcon.2014.05.010. 
[57] Z. Riaz, E. A. Parn, D. J. Edwards, M. Arslan, C. S. Pena-Mora, Feniosky, BIM and sensor-based data management system for BIM and sensor-based data management system for construction safety monitoring, Journal of Engineering, Design and Technolog 15 (6) (2017) 738753. doi:10.1108/JEDT-03-2017-0017.

[58] J. Zhang, B.-c. Seet, T. T. Lie, Building Information Modelling for Smart Built Environments, buildings 5 (2015) 100-115. doi:10.3390/ buildings 5010100

[59] J. Chen, T. Bulbul, J. E. Taylor, G. Olgun, A Case Study of Embedding Real-time Infrastructure Sensor Data to BIM, Construction Research Congressdoi: $10.1061 / 9780784413517.028$.

[60] R. Attar, Ebenezer Hailemariam, S. Breslav, A. Khan, G. Kurtenbach, Sensor-enabled Cubicles for Occupant-centric Capture of Building Performance Data, in: ASHRAE Transactions 117, 2011.

[61] C. Andersson, J. Akesson, C. Fuhrer, PyFMI: A Python Package for Simulation of Coupled Dynamic Models with the Functional Mock-up Interface, Tech. rep., Centre for Mathematical Sciences, Lund University. (2016).

URL pyfmi\{_\}tech.pdf

https://lucris.lub.lu.se/ws/files/7201641/

62] R. Kramer, J. van Schijndel, H. Schellen, Inverse Modeling of Climate Responses of Monumental Buildings, Building and Environment 68 (2013) 87-99. doi:10.1016/j . buildenv. 2013.06.001.

[63] L. Scanu, S. Ploix, P. Bernaud, E. Wurtz, Model Tuning Approach For Energy Management Of Office and Apartment Settings, in: building simulation 2017,Aug 2017, San Francisco, United States., 2017. doi : 10.26868/25222708.2017.233.

[64] F.-A. Fortin, F.-M. De Rainville, M.-A. Gardner, M. Parizeau, C. Gagné, DEAP : Evolutionary Algorithms Made Easy, Journal of Machine Learning Research 13 (12) (2012) 2171-2175 [cited 2018-08-14].

URL http://www.jmlr.org/papers/v13/fortin12a.html

[65] F. Jorissen, G. Reynders, R. Baetens, D. Picard, D. Saelens, L. Helsen, Implementation and verification of the IDEAS building energy simulation library, Journal of Building Performance Simulationdoi:10.1080/ 19401493. 2018.1428361. 